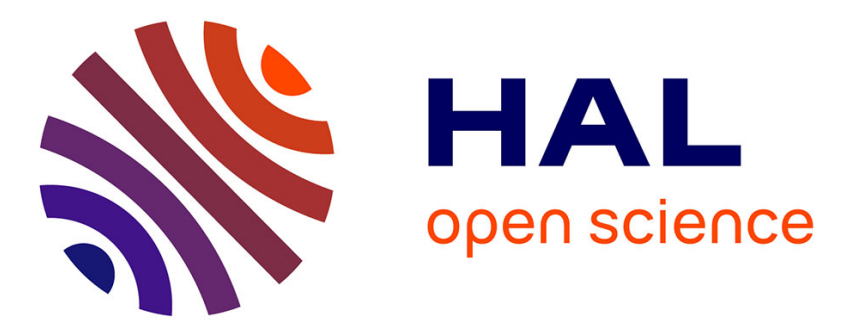

\title{
A semantic-enabled and context-aware monitoring system for the internet of medical things
}

Ahlem Rhayem, Mohamed Ben Ahmed Mhiri, Khalil Drira, Said Tazi, Faiez Gargouri

\section{- To cite this version:}

Ahlem Rhayem, Mohamed Ben Ahmed Mhiri, Khalil Drira, Said Tazi, Faiez Gargouri. A semanticenabled and context-aware monitoring system for the internet of medical things. Expert Systems, 2021, 38 (2), pp.e12629. 10.1111/exsy.12629 . hal-02944704

HAL Id: hal-02944704

https://hal.laas.fr/hal-02944704

Submitted on 19 Nov 2021

HAL is a multi-disciplinary open access archive for the deposit and dissemination of scientific research documents, whether they are published or not. The documents may come from teaching and research institutions in France or abroad, or from public or private research centers.
L'archive ouverte pluridisciplinaire HAL, est destinée au dépôt et à la diffusion de documents scientifiques de niveau recherche, publiés ou non, émanant des établissements d'enseignement et de recherche français ou étrangers, des laboratoires publics ou privés. 


\section{ARTICLE TYPE}

\section{A semantic-enabled and Context-aware Monitoring System for the Internet of Medical Things}

\section{Ahlem Rhayem*1 | Mohamed Ben Ahmed Mhiri ${ }^{1}$ | Khalil Drira ${ }^{2}$ | Said Tazi ${ }^{2}$ | Faiez Gargouri ${ }^{1}$}

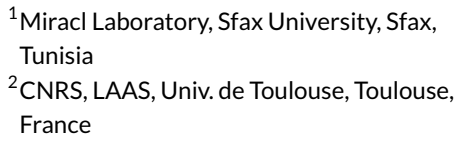

Present Address

Present address

\begin{abstract}
The emergence of the Internet of Things (IoT) in the medical field has led to a myriad of medical connected objects (MCOs). These MCOs are being developed and implemented for remote healthcare monitoring purposes (elderly patient with chronic diseases, pregnant woman, patients with disabilities). Accordingly, different associated challenges are emerging and include the heterogeneity of the gathered health data from these MCOs with ever-changing contexts. These contexts are relative to the continuous change of constraints and requirements of the MCOs deployment (time, location, state). Other contexts are related to the patient (medical record, state, age, sex, and so on) that should be taken into account to ensure a more precise and appropriate treatment of the patient. These challenges are difficult to address due to the absence of a reference model for describing the health data and their sources and linking this data with their contexts. This paper addresses this problem and introduces a semantic-based context-aware system (IoT Medicare system) for patient monitoring with MCOs. In fact, this system is based on a core domain ontology (HealthloT-O) that is designed to describe the semantic of heterogeneous MCOs and their data. Moreover, an efficient interpretation and management of this knowledge in diverse contexts are ensured through SWRL rules such as the verification of the proper functioning of the MCOs and the analysis of the health data for diagnosis and treatment purposes. A case study of gestational diabetes disease management is proposed to evaluate the effectiveness of the implemented loT Medicare system. An evaluation phase is provided and focuses on the quality of the elaborated semantic model and the performance of the system.
\end{abstract}

KEYWORDS:

Medical Connected Objects; Patient monitoring; Ontology; Context-awareness

\section{1 | INTRODUCTION}

It has been revealed that loT enables seamless communication between diverse devices and objects and helps people to interact continuously with them to establish a large connected network. The loT is widely applied especially in the healthcare domain. According to Cai et al. 2014. Fernandez and Pallis 2014 medical care and health care stand as one of the most attractive application areas for the loT.

Referring to the association of important R\&D direction, the loT with the medical sector applications gave birth to an Internet of Medical Things (IoMT11 According to Gatouillat, Badr, Massot, and Sejdić 2018, loMT designates the interconnection of communication-enabled medical-grade devices and their integration to wider-scale health networks in order to improve patients' health. This harmonious relationship paves the way of various intelligent services such as diagnoses and prevention of illness, decision support for appropriate treatment for patients, and remote patient

\footnotetext{
${ }^{1}$ https://www.huffingtonpost.com/josh-stein/the-emergence-of-the-inte_b_6801714.html
} 
monitoring Islam, Kwak, Kabir, Hossain, and Kwak 2015. Moreover, this use of connected objects makes the disease identification easier since its appearance and through its evolution. Therefore, this reveals that loMT changes the medical field from a reactive and fragmented domain that is a hospital and disease-centered to a preventive and interoperable one that mainly focuses on the patient's well-being and quality of life.

In this way, several medical connected objects are developed and deployed such as Zio Patch that measures heart rate and electrocardiogram (ECG) Tung, Su, Turakhia, and Lansberg 2015, smart wearable devices are proposed by Novartis and Google to observe the blood glucose level Senior 2014, Withings is a wireless blood pressure monitor developed by Nokia Topouchian et al. 2014], mobile devices to monitor the progression and treatment of Parkinson disease 22 is an ongoing project between Pfizer and IBM, and so on.

Patients use these MCOs to monitor their measurements (temperature, blood pressure, etc). In fact, through the communication technologies, MCOs are enabled to exchange the detected measurements with the doctor's medical object for helping him for continuous and remote monitoring of their patients. After the diagnosis of the received measures, the doctor can share the result with the patient or with his family. Accordingly, since these MCOs are designed by different manufacturers (Google, IBM, Nokia, etc), they are heterogeneous in terms of deployment contexts, computing capabilities, and communication protocols. Accordingly, the exchanged information is heterogeneous in formats and units and does not have the same coding format. In this context, ensuring the semantic interoperability between these heterogeneous MCOs becomes difficult.

To deal with this challenge a reference model that defines all of the MCOs, their data, and their formats is required to ensure interoperable MCOs. This model should define the MCOs, in terms of their characteristics, capabilities, deployment contexts, measurements and so on. It should also contain knowledge about the observed patient such as symptom, treatment, event, risk.

To this end, Semantic Web Technologies Berners-Lee, Hendler, and Lassila 2001 and especially ontologies are a promising solution. Ontology allows representing knowledge about the domain in a well structured and comprehensive description. According to Studer Studer, Benjamins, and Fensel 1998) an ontology is a formal, explicit specification of a shared conceptualization of a domain of interest. Ontology explicitly defines the loT domain and the healthcare domain knowledge and their relationship.

On the other hand, to ensure the adequate and correct management of the obtained health data, it is primordial to link them with their various contexts. In fact, in our work, we focus on two types of contexts: the patient context and the employed device context. For the first one, the patient health status context can be considered in order to decide the adequate diagnosis and treatment, e.g the blood glucose level of a pregnant woman is different from that of a normal one. In addition, the time context is used to define the required time to monitor the patient and to analyze his health data. Moreover, we can also focus on the location context to determine the place of the patient and make a suitable intervention. Concerning the employed device context, it is required to verify and diagnose its state in order to be sure about the reliability of the gathered data and to be able to easily repair it in case of damage.

From this viewpoint, context-awareness computing Perera, Zaslavsky, Christen, Compton, and Georgakopoulos 2013 is an important research topic that should be taking into account in the loMT field. It allows the interpretation of the patient's health state, avoids misdiagnosis and errors due to the misunderstanding of the patient state by assigning the clinical signs to their contexts.

Hence, the present paper exploits Semantic Web Technology (SWT) Berners-Lee et al. 2001] and context-awareness computing Yürür et al. 2016. and suggests a semantic-based context-aware patient monitoring approach. This approach is composed by four main phases: the data collection and preprocessing phase, the semantic modeling phase, the analysis phase, and the implementation phase.

The main novelties of this paper lie in the following aspects:

- Proposing a unified and standard model (HealthloT ontology) of the obtained data (technical and health data) to be efficiently used by heterogeneous healthcare loT-based systems. This model contains knowledge about the loT and the healthcare domain and the relationships between them. At this level, we aim to extend our previous work on HealthloT in Rhayem, Mhiri, and Gargouri 2017 Rhayem, Mhiri, Salah, and Gargouri 2017, where we proposed a HealthloT ontology, by implementing new concepts related to the contexts of the medical devices and patients respectively.

- Assisting doctors in the exploitation of the collected data by the technique of automated reasoning based on SWT. We consider here the use-case of gestational diabetes context and we develop the related analysis rules that are based on the generic ontology (HealthloT)

- Verifying and configuring the proper functioning of the employed MCO by exploiting its employment context information, in order to guarantee the certainty of the detected data.

- Developing a clinical decision support system (IoT Medicare system) to implement the proposed knowledge base based on SWT technologies (SPARQL, Jena API) for automatic selection of clinical and configuration services. Thereby, at this level, we define end-users interfaces taking into account the requirements of three users (patient, doctor, administrator).

\footnotetext{
${ }^{2}$ https://www-03.ibm.com/press/us/en/pressrelease/49475.wss
} 
- Evaluating the semantic quality of the proposed knowledge base (HealthloT ontology) based on the OQUARE frameworkDuque-Ramos et al. 2014. In addition, we aim to assess the performance of our system in terms of precision, recall, f-measure and response time.

The rest of this paper is organized as follows: Section 2 describes our proposed use case about gestational diabetes monitoring. Section 3 provides an overview about loMT systems and the semantic representation in this domain. Section 4 highlights our semantic-based context-aware architecture, which is composed of four main phases: the data collection and preprocessing, the semantic modeling, the analysis, and the implementation phases. These phases are described in Section 5, 6, 7 and 8 respectively. The evaluation of our approach is illustrated in Section 9 based on technical and functional levels and a comparison with existing approaches. Finally, section 10 draws our conclusion and suggests some future perspectives.

\section{2 | USE CASE: GESTATIONAL DIABETES MONITORING}

Gestational Diabetes is a disease that should be continuously monitored in order to follow its evolution and make an adequate decision according to its behavior. In this context, a pregnant woman needs to communicate and to share her glucose level with the medical staff, receive notification from them, accelerate their intervention in difficult cases. On the other hand, medical staff needs easy access to the patient's data to interpret them and suggest the appropriate treatment. To alleviate these challenges, the medical sector adopts loT technology. Thereby, various researchers Cappon, Acciaroli, Vettoretti, Facchinetti, and Sparacino 2017, Kim, Campbell, and Wang 2018, Wang and Lee 2015. have implemented diverse MCOs to monitor the state of a pregnant woman. These MCOs contains diverse sensors to detect the patient's blood glucose, heartbeat, blood pressure, etc. These objects are heterogeneous in terms of deployment contexts, computing capabilities, and communication protocols. They generate a huge amount of heterogeneous and ambiguous data that describe various cases. The health data represents the state of the patient and technical data concerning the state of the medical objects. They generate a huge amount of heterogeneous and ambiguous data that describe various cases. The health data represents patients' states while technical data shows the states of medical objects. Moreover, this amount of data, including semantic heterogeneity (synonymy, antonyms, polysemy, etc.), keeps growing. This numeric information can be badly understood and exploited by users. For instance, if the blood glucose sensor value reaches 200 , doctors in this case will need further details to understand that this value refers to the blood glucose level of an ordinary diabetic patient or a pregnant woman that equals to $200 \mathrm{mg} / \mathrm{dl}$. Consequently, information related to the context of the obtained measurements (source, the type of measurement, unit, patient's personal information, time of detection, location and so on) are primordial in both analysis and treatment phases within a reasonable period.

Both doctors and patients use connected objects that are designed by different manufacturers (Google, IBM, Nokia, etc). Accordingly, the generated data has different coding formats leading to a complex data exchange task.

In the same vein, technical data is incredibly important to configure and manage the state of the used object in order to repair any functional breakup.

Assuming that the MCO of a pregnant woman fails (discharged battery, ending lifetime, broken connection), the medical staff encounters a big challenge to remotelymonitor their patient. In this case, these devices should be replaced with anotherof the same type (e.g. blood glucose sensor should not be replaced with a temperature sensor). Hence, the monitoring task will be assigned to the new available object to perform this new task. Indeed, this object should be connected to the internet. Therefore, in indoor environments, the object can be connected to the home gateway as the patient stays at home. In outdoor environments, however, the object can be connected to the internet via the 4G wireless network. In this respect, verifying the connectivity and availability of this object is a vital stepbefore assigning the new task. Besides, testing the MCOs mobility can determine the location of the pregnant woman and consequently facilitate the intervention of healthcare professionals in any emergency case. As a result, treating information about the crossed trajectory is vital to track the MCO mobility. Once the state of the object is reliable, doctors are able to treat and diagnose patients' states. First, the validity of the obtained blood glucose level for possible treatment should be checked. Diagnosing the blood glucose level during a predefined time interval helps avoid adverse events, like fainting and dyspnea. Second, doctors will diagnose blood glucose values in pregnancy. Thus, they are permanently diagnosed during the second and third semesters of pregnancy. These values can be recognized based on one of these two different glucose tests. The Fasting Plasma Glucose (FPG) should be between $92 \mathrm{mg} / \mathrm{dl}$ (5.1 mmol/L) and 126 $\mathrm{mg} / \mathrm{dl}(7.0 \mathrm{mmol} / \mathrm{l})$. The Oral Glucose Tolerance Test (OGTT) is greater than or equals to $180 \mathrm{mg} / \mathrm{dl}(10.0 \mathrm{mmol} / \mathrm{L})$ after one hour and greater than or equals to $153 \mathrm{mg} / \mathrm{dl}(8.5 \mathrm{mmol} / \mathrm{L})$ after two hours according to Association et al. (2018). Third, the diagnostic test results and medical records of a pregnant woman (age, weight, historical diseases, tests) help doctors estimate possible health complications and the most dangerous risks. In fact, according to the World Health Organization (WHO, Organization et al. 2016), uncontrolled diabetes during pregnancy cause hazardous effects on both the mother and child, such as the risk of fetal loss, congenital malformations, and stillbirth. Diabetes treatment in pregnancy depends on the blood-glucose level. In fact,at a medium level, doctors can resort to just a dietary plan. However, at a high glucose level, doctors suggest a medication therapy (for example: metformin 3 times per day during or after meals, insulin injections) that will be taken only during the pregnancy period. After that, doctors send notifications of adequate treatments for patients or their families through alarms sent by doctors'connected objects. In an emergency case, when symptoms of fetal loss appeared, an emergency alert should be forwarded to the hospital in order to urgently send an ambulance. 
To treat these cases, it is essential to develop an application in order to assist users to control and configure MCOs regardless of their location (e.g. a doctor at home can control the remotely-connected medical devices of his patients to verify their reliability and trust; he can send/receive notifications to/from them). Besides, this application will enable users to share knowledge about the state of patients and the appropriate treatments, via their objects. In this regard, the main goal of this work is to propose a semantic-enabled context-aware approach that consists in: - Defining a semantic representation of medical objects with their data and contexts to resolve the semantic heterogeneity problem.

- Facilitating the diagnosis of the health states of pregnant women based on the semantic model by taking into account myriad contexts such as, age, symptoms, hypertension, etc.

- Ensuring the good functioning of the employed medical objects to guarantee sustainable and effective pregnant women's monitoring.

- Facilitating the interaction between doctors and users through the development of a semantic-based patient monitoring system. This system fulfills the following users' requirements:

- Patient requirements: Patients (pregnant women) can continuously control their states by consulting the collected measurements. Each patient is also willing to receive and verify any notification sent by his/her doctor.

- Doctor requirements: The doctor is able to monitor a group of patients by analyzing their measurements in a given context; they can remotely consult the proposed diagnosis report by our system and to make a change if necessary. In addition, doctors can communicate and sent the appropriate diagnosis and results for patients through the triggering of alarms.

- Administrator requirements: The administrator can control the proper functioning of the medical connected objects used by the doctors and their patients. He is able to send notifications for users if he encounters a problem in the object and to automatically resolve it.

\section{3 | LITERATURE REVIEW}

This section is divided into two sub-sections. In the first one, we give an overview of the most recent applications of the loT in the healthcare domain. It is divided into two parts, namely monitoring system for loMT and context-aware monitoring system for loMT. In the second sub-section, we will focus on the semantic representation approaches in this domain. First, we study the proposed approaches in the loT in general. Second, we interest in approaches that define the semantics of loT-based healthcare systems. After that, we underline the main contributions of our work.

\section{1 | loT-enabled Smart Healthcare}

The continuous need of a low-cost remote monitoring system for elderly patients with chronic diseases has encouraged software developers to adopt loT technologies in the healthcare field. The works of Mishra and Rasool 2019] and Jayatilleka and Halgamuge 2020 have overviewed the recently developed loT devices that guarantee a reliable and secure patient monitoring and treatment delivery in the healthcare domain.

This adoption shows important and adequate results for improving the quality of healthcare services in smart environments Turcu and Turcu 2019], Meigal, Korzun, Gerasimova-Meigal, Borodin, and Zavyalova 2019.

In the following sub-sections, we will start by displaying the most recently proposed monitoring system in the loMT. Then, we will focus on the context-aware monitoring system in loMT.

\subsection{1 | Monitoring System for the loMT}

IoT has been widely used for remote monitoring of cardiovascular diseases Jabeen et al. 2019. In fact, diverse applications have been developed for diabetes Puri, Kumar, Le, Jagdev, and Sachdeva 2020, heart disease Khan 2020, hypertension Sood and Mahajan 2018, Alzheimer Varatharajan, Manogaran, Priyan, and Sundarasekar 2018, epileptical patient monitoring Gupta, Chakraborty, and Gupta. 2019], and so on. In what follows, some recent loT-based monitoring systems are detailed.

Diabetes monitoring: The authors in Chatterjee et al. 2018. implemented an loT-based home monitoring system for diabetic patients. This approach aims at monitoring the daily activities of these patients to trigger alerts concerning their dietary behavior when necessary. Puri et al. 2020 have developed an loT-based diabetes monitoring system that is capable of detecting and analyzing blood sugar, body temperature and other environmental parameters (temperature, humidity). This system encrypte data to ensure its secure transmission, analysis and storage in the cloud. Heart rate monitoring: Liu, Chen, and Wang 2018 have applied a medical loT system based on wireless sensor networks for ECG monitoring. This system detect and transmit physiological parameters and image data to the base station. This data can be easily accessed and analyzed by medical professionals through their PDA and computers to ensure a timely treatment in an abnormal state. Therefore, Khan 2020 have developed and loT framework for heart disease prediction. They used a smartwatch and a heart monitor device to monitor blood pressure and electrocardiogram 
measurement. These measurements were then analyzed through the modified deep convolutional neural network (MDCNN) for heart disease prediction. Santos, Trujillo, Portilla, and Rosales 2019] have set forth an e-health system for heart rate monitor in Ecuador. This system supports continuous care and suggests primary diagnostic assistance service of heart disease. It bases its operation on video processing algorithms, which are collected through web cam and mobile device that communicate with a central data base station via WAN network.

Hypertension monitoring: Sood and Mahajan 2018 proposed an loT-fog health monitoring system to monitor the blood pressure level to predict real-time hypertension event. In addition, this system focused on predicting health complications and risks based on several machine learning algorithms and alerts generation.

CoronaVirus monitoring: with the CoronaVirus outbreak, which first appeared in China, several researchers are working on loT exploitation to be able to control and prevent this pandemic. Bai et al. 2020 have offered a diagnosis and treatment system for COVID-19 based on the loT. The main asset of this system is to remotely monitor outpatients in the containment zone and ensure a fast and reliable intervention if necessary. It helps doctors, at the early stages of this disease, guarantee timely treatment of confirmed cases and facilitate the interaction between experts and managers to deeply investigate or expand the diagnosis and treatment of COVID-19. Both a smartphone and a PC, key components of this system, are used for patient monitoring and cloud computing for data storage, communication, and transmission technologies.

In Table 1 we present a comparison of the above-mentioned works, related to the loT-based healthcare monitoring system. This comparison is based on diverse criteria to give a general overview of the main properties of loT-based systems in the healthcare domain. The used devices criterion aims to list the different deployed loT devices. The communication technology identifies the types of the used technologies to connect the IoT devices. The application criterion determines the application domain of the developed system. The use of the ontologies criterion verifies if the proposed works addressed the semantic-interoperability challenge or not. The reasoning methods criterion identifies the principal used methods and technics for data interpretation. The tools criterion verifies if the proposed works have developed and evaluated their approaches on a real use case.

TABLE 1 A comparison of recent monitoring systems for the loMT.

\begin{tabular}{|c|c|c|c|c|c|c|}
\hline References & IoT devices & $\begin{array}{l}\text { Communication } \\
\text { Technology }\end{array}$ & Application & $\begin{array}{l}\text { Use of } \\
\text { ontologies }\end{array}$ & $\begin{array}{l}\text { Reasoning } \\
\text { methods }\end{array}$ & Tools \\
\hline $\begin{array}{l}\text { Chatterjee et } \\
\text { al. 2018 }\end{array}$ & $\begin{array}{l}\text { Ambient sensors (switch, sen- } \\
\text { sor for activity), loT devices (BG } \\
\text { monitor), Body wearable devices }\end{array}$ & WSN & Diabetes & NO & $\begin{array}{l}\text { replicator neu- } \\
\text { ral network } \\
\text { (RNN) }\end{array}$ & Yes \\
\hline$\frac{\text { Puri et al. }}{2020}$ & $\begin{array}{l}\text { Glucose sensor, transcon- } \\
\text { ductance amplifier, and } \\
\text { microcontroller }\end{array}$ & WiFi & Diabetes & NO & If-Then rules & Yes \\
\hline Liu et al. 2018 & ECG sensor & $\begin{array}{l}\text { Wireless Sen- } \\
\text { sor Networks } \\
(\text { WSN) }\end{array}$ & Heart & NO & $\begin{array}{l}\text { image pro- } \\
\text { cessing } \\
\text { techniques }\end{array}$ & Yes \\
\hline Khan 2020 & $\begin{array}{l}\text { smart watch and heart monitor } \\
\text { device }\end{array}$ & $\begin{array}{l}\text { LoRa } \\
\text { technology }\end{array}$ & Heart & NO & $\begin{array}{l}\text { Modified Deep } \\
\text { Convolutional } \\
\text { Neural Network } \\
\text { (MDCNN) }\end{array}$ & Yes \\
\hline$\frac{\text { Santos et al. }}{2019}$ & Web cam and & WAN network & Heart & NO & & \\
\hline $\begin{array}{l}\text { Sood and } \\
\text { Mahajan } \\
2018\end{array}$ & $\begin{array}{l}\text { Health sensor (Blood pres- } \\
\text { sure sensor, heart arte; etc), } \\
\text { accelerometer, Bio sensor, RFID } \\
\text { tag, etc }\end{array}$ & & Hypertension & NO & $\begin{array}{l}\text { Artificial Neural } \\
\text { Network (ANN) }\end{array}$ & Yes \\
\hline Bai et al. 2020 & $\begin{array}{l}\text { sensors for heart rate, tempera- } \\
\text { ture, respiratory rate, etc }\end{array}$ & 5G technology & Coronavirus & NO & & Yes \\
\hline
\end{tabular}




\subsubsection{Context-aware Monitoring System for the loMT}

To ensure reliable and efficient health data analysis and interpretation, an loT-based system should understand the contextual information of the obtained measure. Dey, Abowd, and Salber 2001) have defined context as "any information that can be used to characterize the situation of an entity. An entity is a user, place, or physical or computational object that is considered relevant to the interaction between a user and an application, including the user and applications themselves". Perera et al. 2013] and Yürür et al. 2016) have surveyed context-awareness from an loT perspective.

In this section, we propose an overview of the suggested loT-based context-aware systems.

Rahman, Rahmani, and Kanter 2017] have put forward a multi-modal context-aware reasoner (CAN) for different loT applications at the loT edge. First, contextual information will be extracted from raw data. Second, this information will be filtered and then transfered to the reasoner to decide to which application it may refer.

Chegini and Mahanti 2019 have defined a microservice framework to implement automatic functionalities for loT-fog-cloud ecosystem, such as automatic loT orchestration and collaboration, automatic task workflow, automatic task scheduling, etc. This framework helps developvloT-based context-aware intelligent decision-making systems.

To tackle the challenges of heterogeneous data fusion and context-awareness query processing in the loT health domain, Baloch, Shaikh, and Unar 2018 have proposed a context-aware data fusion in the loMT domain. This approach is composed of context acquisition, data fusion, and inference and reasoning steps.

Authors in Aborokbah, Al-Mutairi, Sangaiah, and Samuel 2018, have also introduced an SVM-based context-aware decision support system for healthcare service delivery in smart cities. This system interpreted the patient's clinical state based on multiple vital signs (heart rate, temperature, blood pressure) for early prediction of heart failure risks. Furthermore, it could provide real-time analysis of physiological data to continuously determine the state of the patient and provide optimal health care services.

Barbosa, Tavares, Cardoso, Alves, and Martini 2018 have offered a context-aware system to assist wheelchair users. It recommends accessible resources in indoor and outdoor environments during their displacement process based on various contexts, like location, time, users' identity, etc. To ensure the security of health data transmission between sensors, Arfaoui, Kribeche, and Senouci 2019 have adopted a context-aware and lightweight anonymous authentication scheme for Wearable Body Area Networks (WBAN) applications in emergency and normal situations. Their proposed scheme provides selective anonymous authentication between nodes in WBAN while taking into account the dynamic context changes (the battery level of sensors, memory capacity, etc).

Table 2. shows a comparative study of the context-aware monitoring systems. We are based on the same criteria as the previous table and we add another one concerning the type of the treated contexts with the aim to highlight the most important contexts processed by these works.

According to Table 1 and Table 2, diverse types of loT devices are used in the healthcare domain and connected to various communication technologies. In addition, they are based on diverse techniques for data interpretation and management tasks. However, approaches in Table 1 put special focus on context-awareness in the internet of medical things. Several contexts are defined, such as location, time, patient's state, device's requirement, etc. In fact, contexts related to the device's functioning are only considered by Arfaoui et al. [2019], who directed their attention to ensure safe operation of loT devices during data collection and transmission.

Moreover, approaches presented in Table 2 and 3 have not considered using ontologies to model and manage their contexts and to deal with the semantic interoperability problem in the loMT domain. This was our main objective in this paper. From this regard, much attention has been given to the related works presented in section 3.2

\subsection{Semantic Representation in the loMT domain}

In this section, we will focus on the semantic representation of the loMT knowledge. Accordingly, it is crucial to study at first the proposed approaches in the loT field in general and then on the loMT.

\subsection{1 | Internet of Things Ontologies}

Over the last few years, diverse ontologies are suggested in the loT field. The most referenced work was suggested by Compton et al. 2012, who put forward a Semantic Sensor Network (SSN) ontology. It describes sensors in terms of capabilities, measurement processes, observations and deployments in order to define the semantic interoperability of physical sensor networks. Its core concepts are sensors and their properties, 
TABLE 2 Comparison between Context-aware Monitoring systems for the loMT

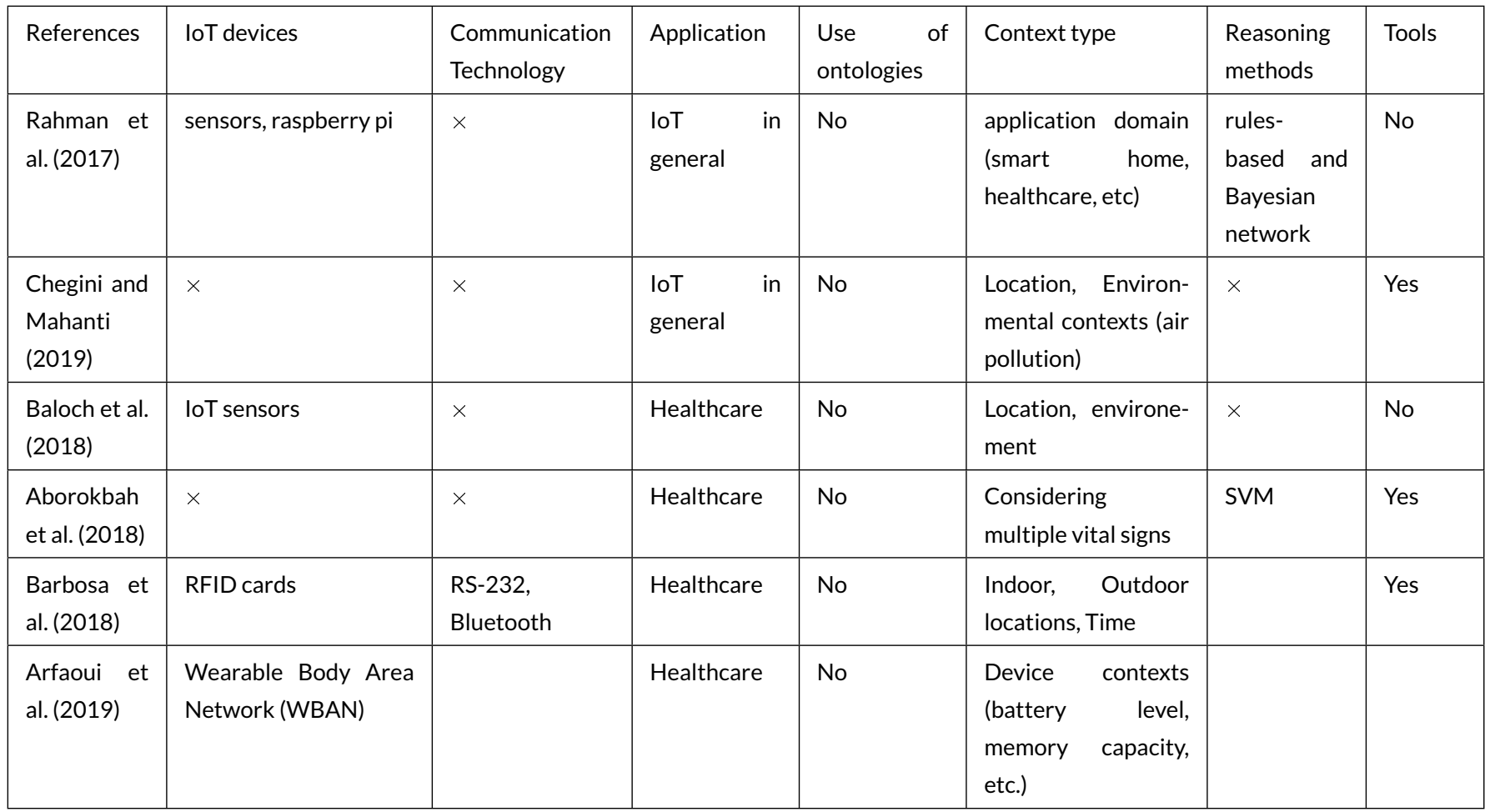

observations, systems, measuring capabilities, operational and survival restrictions, and deployments.

The SSN ontology is then extended to wireless sensor networks (wireless sensor network ontology) by Bendadouche, Roussey, De Sousa, Chanet, and Hou 2012. It is also used later to model sensor clouds (sensor cloud ontology) by Müller, Cabral, Morshed, and Shu 2013] and multimedia sensor by Angsuchotmetee, Chbeir, and Cardinale 2018.

Nonetheless, the loT domain does not only include sensors, but also other concepts that have to be addressed, namely the actuator, the physical object. The former is responsible for generating actions while the latter is connected to the internet and integrates sensors, actuators, devices, among others. For that reason, diverse approaches were realized in the last few years in order to represent the loT domain knowledge. Among these approaches, we will present the most common and cited ones.

To achieve this purpose, Bauer et al. 2013] have defined an loT-A architecture in loT. This model defines the core concepts of this domain, such as physical entity, virtual entity, sensor, tag, actuator, service, and user.

In addition, to represent the semantics of actuators and their capabilities and roles, the SOSA ontology was suggested by Janowicz, Haller, Cox, Le Phuoc, and Lefrancois 2018. It was proposed by both the joint group World Wide Web Consortium (W3C) and the Open Geospatial Consortium (OGC) in order to elucidate the interactions between sensors, observations, actuators and sample concepts.

Therefore, in the context of the ADREAM project, Seydoux, Drira, Hernandez, and Monteil 2016, proposed a modular ontology (IoT-O 3 to ensure a semantic interoperability between loT components. loT-O contains several modules, like sensing, actuation, life cycle, service and energy modules. Ma, Wang, and Chu 2014] set forth a semantic information model for loT applications, known as OntoloT ontology. In fact, the latter describes (i) real word entities (objects being monitored, sensor devices, and network infrastructure), (ii) spatial and temporal dimensions, (iii) the captured (dynamic and static) data, (iv) services including applications (e.g. in the areas of healthcare or traffic), functions, and interfaces.

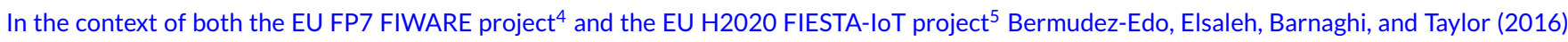
developed an loT-lite ontology with the aim of describing loT concepts in three different classes: Objects, systems or resources, and services. This ontology is a lightweight instantiation of the SSN ontology.

In the same context, Agarwal et al. 2016 proposed a unified ontology, which reuses a number of core concepts from several ontologies, such as Semantic Sensor Network (SSN), M3-lite, WGS84, loT-lite, Time, and DUL in the loT domain.

\footnotetext{
${ }^{3}$ https://www.irit.fr/recherches/MELODI/ontologies/loT-O/

${ }^{4}$ https://www.fiware.org/

${ }^{5}$ http://fiesta-iot.eu/
} 
TABLE 3 Comparison of Semantic-loT related works

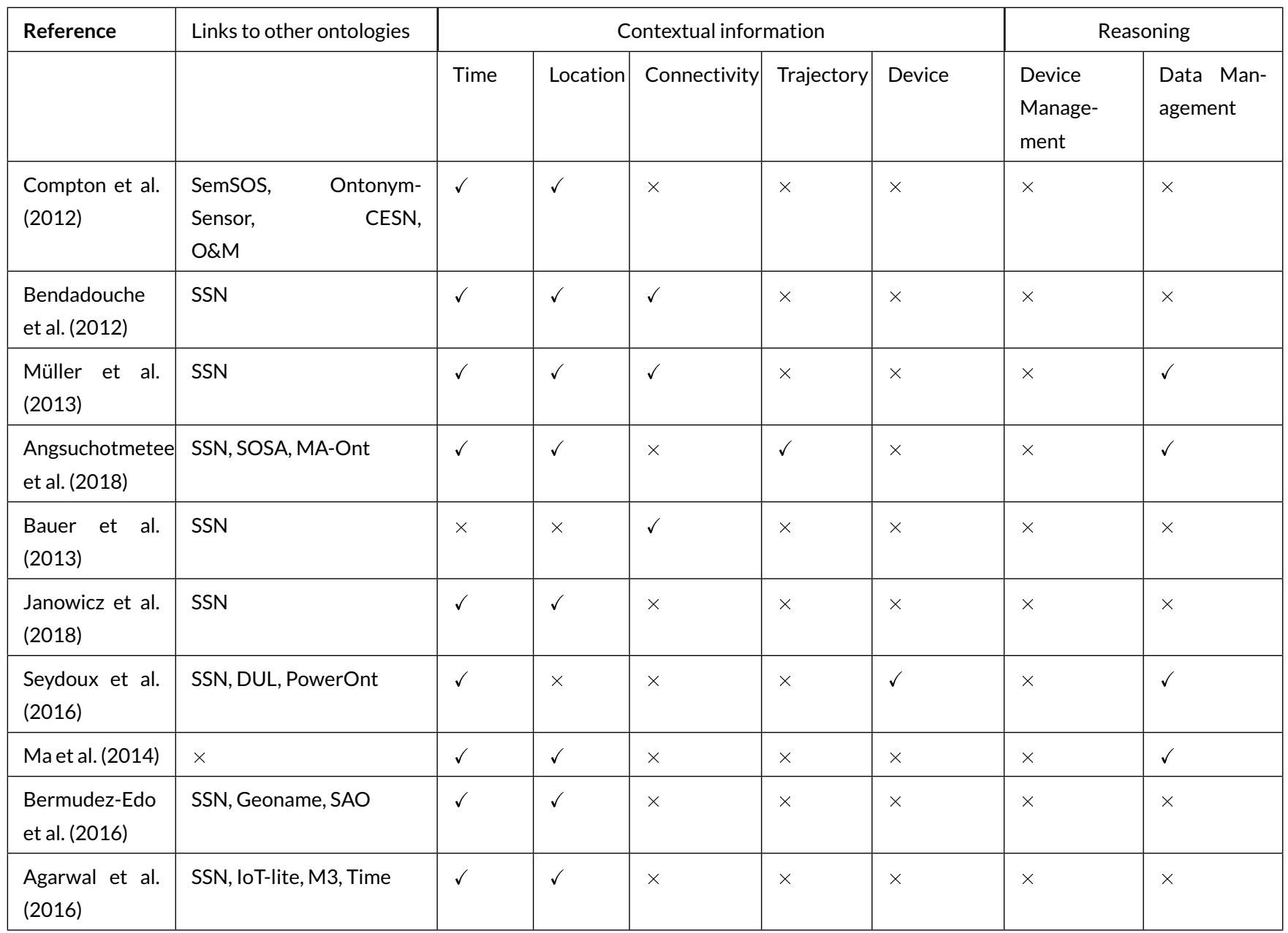

We present a comparative table, of the different approaches mentioned above, that shows the use of ontologies to resolve the semantic heterogeneity in the loT domain. The comparison is based on the following criteria:

- Links to other ontologies: within this criterion, we aim to identify if the proposed ontology reuses concepts from a previous one and to check whether these ontologies are built from scratch.

- Contextual information: This criterion determines the presented contexts in the proposed ontologies. We have identified five major contexts that are important in the loT domain, namely time, location, inter-connectivity, trajectory and objects' requirements.

- Reasoning: This criterion verifies whether the proposed approaches suggested rules for either device management or data management.

According to Table 11 we notice that most of these approaches take advantage of reusing existing ontologies instead of building new ones from scratch. SSN was one of the most referenced ontologies in these approaches as it presents a pivotal component for each loT-based system. Furthermore, the main modeling contexts in these works are time and location with little emphasis on interconnectivity, requirement, and trajectory contexts. Concerning the reasoning phase, none of these approaches was interested in managing the state of the employed object, such as checking its availability, connectivity, and task allocation. Therefore, reasoning about the obtained data from loT devices was addressed by only some works, like Müller et al. 2013, Angsuchotmetee et al. [2018], Seydoux et al. 2016, Ma et al. 2014].

The next sub-section will be devoted to the semantic representation of the adoption of loT in the healthcare domain.' 


\subsection{2 | Semantic-based loMT systems}

Ensuring the semantic interoperability in the loT and the healthcare fields gave rise to diverse research issues. Therefore, numerous ontologies are available in the medical domain. SNOMED-CT6 is the most known thesaurus, in which medical terms and their synonyms, are treatable with machines.

The Open Biomedical and Biological Ontology (OBO), according to Smith et al. 2007, was developed in the field of biomedical informatics. It is based on the upper-level ontology Basic Formal Ontology (BFO).

UFO (ECG) ontology Gonçalves, Zamborlini, and Guizzardi 2009] is an ontology for the electrocardiogram diagnosis based on the UFO ontology. This model proposed a reference and a standard model of the ECG data.

Therefore, several researchers have addressed the semantic interoperability issue in the loMT domain. Authors in Lasierra, Alesanco, O'Sullivan, and García 2013. provided an ontology representing the management procedure MAPE-Loop (monitoring, analysis, planning, and execution) of technical data in the case of a patient monitoring.

Authors in Alirezaie et al. 2017 came up with an ontology-based system called "E-care@home". It consists of three different parts, namely Ecare@home database, loT devices, and software and protocols. This Smart Home Ontology is proposed to describe and interpret the heterogeneous sensor data.

The work achieved by Forkan, Khalil, and Tari 2014 proposed a cloud-based real-time context-aware framework in ambient assisted living. This framework deals with the aggregation and management of heterogeneous sensor data in different contexts. In D. Chen, Jin, Goh, Li, and Wei 2016, the authors proposed an Antihypertensive Drugs Personalized Recommendation Service Context Ontology (HyRCO). This model is divided into seven core classes, namely: User, Activity, Environment, Device, Service, Location and Anti-hypertensive. It defines context rules to provide a drug recommendation service for the hypertension disease based on SWRL language. Recently, the SAREF ontology was extended for the healthcare domain Moreira, Pires, van Sinderen, and Daniele 2018] and specifically for monitoring ECG data.

Esposito et al. 2018 have implemented an ontology-based context-aware architecture for personal monitoring that can be deployed in mobile devices. This architecture has addressed the challenge of self-configuring loT devices, contextual information extraction related to patients for later analysis and interpretation, the fusion of contextual information with sensor data in order to detect suspicious anomalies and supply adequate alerts. This architecture comprises four distinct layers, namely the sensing layer, the perceptual layer, the reasoning layer and the actuating layer. Rubí and Gondim 2020, have developed an interoperable loMT platform by the alignment between the SSN ontology and the Electronic Health Record (EHR). This platform is based on the M2M architecture that enables communication between the different components of the loT platform. Table 4displays a comparison of the developed ontologies in the loMT domain based on some criteria. First, the loT devices criterion determines the modeled loT devices in the proposed ontologies. Second, the interoperability criterion verifies whether the proposed ontology was either developed from scratch or based on reusing other ontologies. Third, the context-aware criterion demonstrates if the ontology contains concepts related to both the deployed medical context, such as time, location, and trajectory, and patients' contextual information, like diseases, symptoms, historic data. Fourth, the reasoning criterion determines whether the proposed works were interested in not only diagnosing the patients' state, anticipating possible risks for prevention purposes, and proposing treatment but also checking the connected objects' states. Finally, the users' criterion aims to identify to whom the proposed system was addressed.

\section{3 | Synthesis}

After examining Table 4, we can still recognize some shortcomings in applying SWT in loMT, as described below.

- None of the aforementioned works proposed an ontology that covers the essential concepts in both the loT and Healthcare domains. The majority of the suggested approaches in the healthcare field ignored reusing the already defined loT models. That is to say, they limited health data source description to only one sensor concept.

- Little attention was paid to the semantic relationships between loT and healthcare components that can provide a comprehensive model to analyze health data gathered from loT devices.

- The semantic context modeling is limited to time, location and users in the almost of approaches. It does not represent a specific context (e.g. capability, network, trajectory) for medical connected objects that affects their functioning. In addition, health care contexts were not considered in these approaches.

\footnotetext{
${ }^{6}$ http://www.snomed.org/
} 
TABLE 4 Criteria and Health care ontologies comparison

\begin{tabular}{|c|c|c|c|c|c|}
\hline $\begin{array}{l}\text { References } \\
\text { Criterion }\end{array}$ & loT devices & Interoperability & Context-aware & Reasoning goals & Users \\
\hline $\begin{array}{l}\text { Lasierra et al. } \\
2013\end{array}$ & sensors & No & No & $\begin{array}{l}\text { Device management: ON, } \\
\text { OFF }\end{array}$ & Patient \\
\hline $\begin{array}{l}\text { Alirezaie et al. } \\
2017\end{array}$ & sensors & SSN & $\begin{array}{l}\text { Time, Loca- } \\
\text { tion, Patient's } \\
\text { activity }\end{array}$ & Activity recognition & Patient \\
\hline Forkan et al. & sensors & No & $\begin{array}{l}\text { Device, Envi- } \\
\text { ronment, Place, } \\
\text { Person }\end{array}$ & Diagnosis & Patient \\
\hline $\begin{array}{l}\text { D. Chen et al. } \\
2016\end{array}$ & sensors & No & Patient & $\begin{array}{l}\text { Treatment for hypertension } \\
\text { disease }\end{array}$ & Patient \\
\hline $\begin{array}{l}\text { Moreira et al. } \\
2018\end{array}$ & sensors & $\begin{array}{l}\text { SSN/SOSA, UFO } \\
\text { ECG }\end{array}$ & No & No & Patient \\
\hline $\begin{array}{l}\text { Esposito et al. } \\
2018\end{array}$ & sensors & No & $\begin{array}{l}\text { Patient's } \\
\text { activity }\end{array}$ & Diagnosis & $\begin{array}{l}\text { Doctor and } \\
\text { Patient }\end{array}$ \\
\hline $\begin{array}{l}\text { Rubí and } \\
\text { Gondim } \\
2020\end{array}$ & Sensors & SSN & No & No & Doctor \\
\hline
\end{tabular}

- The reasoning task is conceived to analyze the patient's health care state in a simple case. Researchers have not treated contexts in a detailed way. Additionally, to the best of our knowledge, none of these approaches have been interested in managing and interpreting the states of medical connected devices.

- Except for the study in Y. Chen, Zhou, and Guo 2016, all the proposals do not take into account the whole diagnosis process which starts by detecting health data from heterogeneous sources followed by analyzing the occurring event, then, anticipating risks and finally suggesting the suitable service in order to provide real time notifications to patients.

From this perspective, our purpose was to address these problems. First, in order to increase the interoperability of our ontology, we reused some concepts extracted from diverse loT ontologies, such as SSN, IoT-O, and loT-lite. Second, diverse contexts were presented in our model that deals with the functioning of the used objects and the patients' states. Third, in the reasoning step, we focused on the remote monitoring of both medical objects and patients. To this end, we put forward and emphasized the M-E-R-T-A process which started by analyzing the obtained data with a special emphasis on other factors (symptoms, historical diagnosis, age, sex), predicting the potential health risks and finished with the treatment proposition.

\section{4 | SEMANTIC-BASED CONTEXT-AWARE APPROACH FOR IOMT}

In this section, we detail our proposed semantic-based approach as depicted in Figure 1 for patient monitoring through MCOs. This approach is based on four mains phases. The first phase describes the data collection process via the MCO and the patient medical records. In order to understand this data, a semantic modeling representation is suggested by defining their sources and their contexts in relation with either the MCO or patient. As a result, we obtain a HealthloT ontology that will be analyzed by proposing three categories of SWRL rules. The first ones are used to configure and manage the MCO function state. The second ones are highlighted to diagnose the patient state and to predict possible health complication risks. The last category is defined to notify end-users by sending suitable alerts. These three previous phases represent the knowledgebase that will be exploited in our implementation phase to provide the appropriate services for end-users through a friendly user interfaces. These phases will be well detailed in the next sections. 


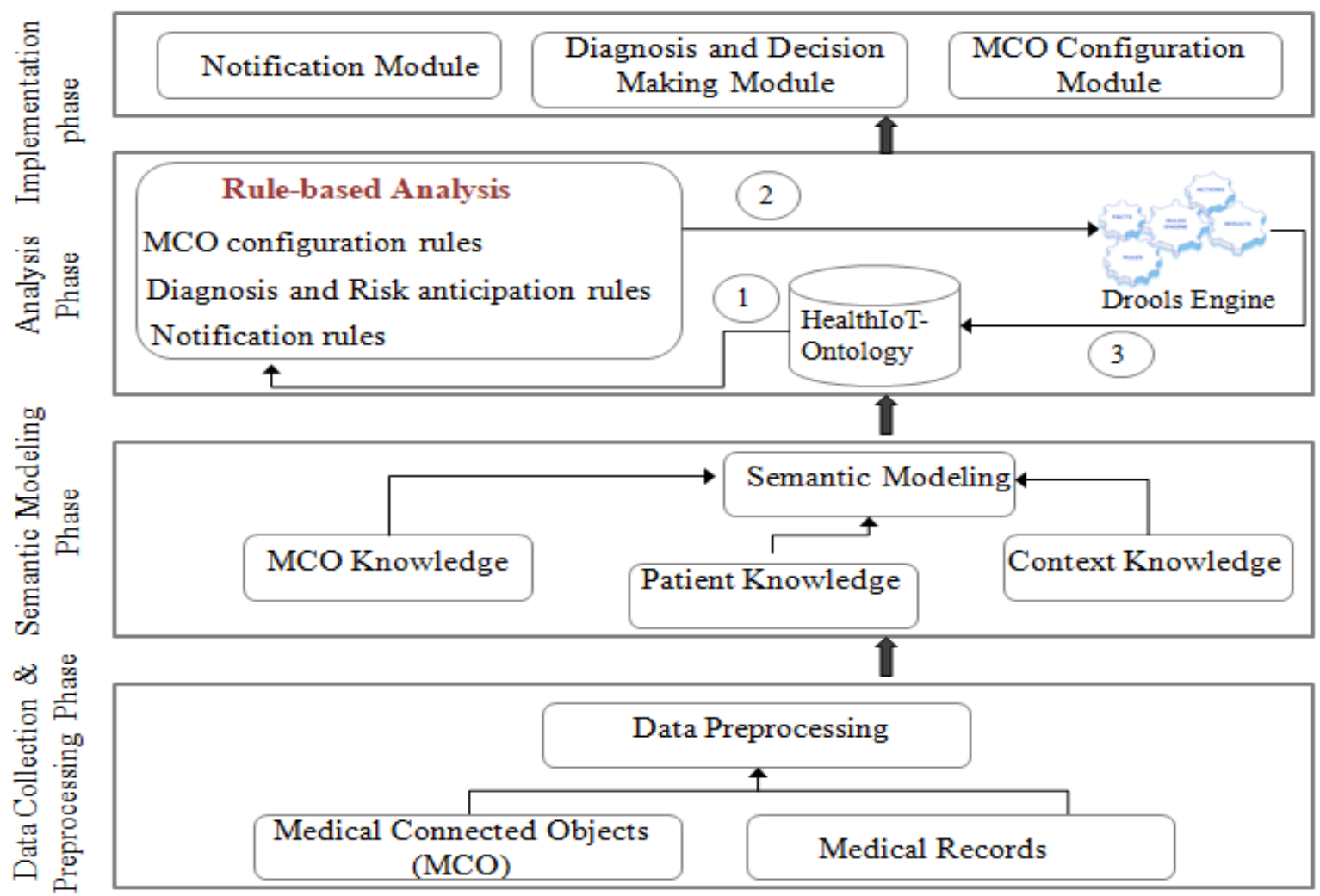

FIGURE 1 Semantic-based context-aware approach

\section{5 | DATA COLLECTION AND PREPROCESSING PHASE}

Heterogeneous health data is required to understand more the medical system features. Accordingly, comprehensible and reliable data analysis will be easily performed that enables seamless and efficient communication between healthcare professionals and patients. In our work, we use two different data sources such as medical connected objects and medical reports that provide dynamic and static health data, respectively.

\section{1 | Data from medical connected objects}

In managing and monitoring gestational diabetes, doctors pay attention to patient information that comes from several sources. Blood glucose monitoring devices are exploited for continuous monitoring of blood glucose levels. Therefore, to avoid health complications during pregnancy, diverse other measurements should be taken into account, such as blood pressure, heart rate, cholesterol, patients' activity. In this context, patients consider other medical objects, namely ECG monitoring devices, blood pressure monitoring devices and smartphones containing sensors for activity tracking. Furthermore, according to Abu-Elkheir, Hayajneh, and Ali 2013, data captured through connected objects are classified into two distinct categories: technical data which describes the general context of the data collection process (time, Id sensor, space, MCOs properties, etc.) and data relative to the monitored patient (measurement, unit of measurement, etc). Figure 2 represents an example of temperature data obtained from medical object in JSON format, where the red color designates the technical data and the blue color designates the health data. In this study, we use various data sets (CSV format) from the physiobank 7 namely temperature, cholesterol, blood pressure, blood glucose, and heart rate. PhysioBank is a large and growing archive of well-characterized digital recordings of physiologic signals and related data for use by the biomedical research community. It is one of the resources of the National Institutes of Health 8 which is intended to stimulate current research and new investigations in cardiovascular studies and other complex biomedical signals Goldberger et al. [2000. These data sets contain numeric and string data type.

\footnotetext{
${ }^{7}$ https://physionet.org/data/

${ }^{8}$ https://www.nih.gov/
} 


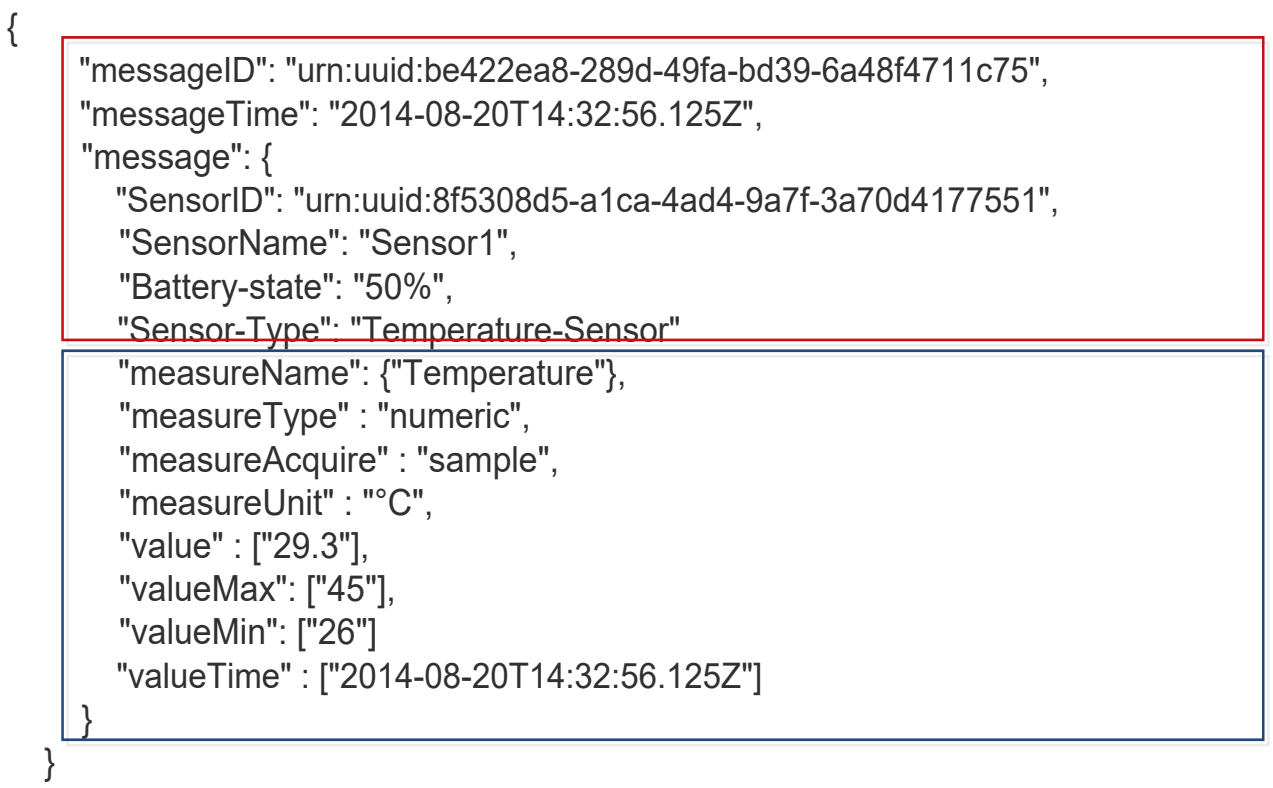

FIGURE 2 Obtained data from sensors

\section{2 | Data from medical rapport}

To provide a precise and suitable treatment for patients, doctors should not only rely on data from mobile objects. In this regard, they need to refer to patients' medical records as another source of healthcare data. This source contains basic patient information (age, sex, name, address), laboratory test data, symptoms, patient medical record, and medical family history. In our work, these valuable information are provided by three domain experts (doctors) from their patients' personal health records (PHR). In that phase, the SNOMED-CT terminology is recommended by our experts to represent this clinical information as it contains the largest clinical terminology.

\section{3 | Data Preprocessing}

Due to the ambiguous, heterogeneous and noisy data acquired from the MCOs, a preprocessing phase should be performed to reduce and remove erroneous and unusual data. First, we apply a data cleaning phase that consists in removing data with values outside the loT devices thresholds. In fact, each device has a maximum and a minimum range. Healthcare data with values outside this range are considered as erroneous data that will be removed. Missing data are also removed. Second, we carry out a normalization phase before integrating this data in the ontology to avoid such inconsistencies. For example we have changed the time format (from "YYYY/MM/DD hh:ss:mm" to "YYYY-MM-DDThh:ss:mm") using the ISO 8601 standard adopted by SNOMED-CT.

In order to make this data understandable and interpretable by MCO and loMT-systems, the next section will be devoted to the formal representation of the MCO and the healthcare domain knowledge and their relationships. The OWL2 Motik et al. 2009) is used for the formalization of the knowledge domain.

\section{SEMANTIC MODELING PHASE}

In this phase, a HealthloT ontology Figure 3 is proposed for the following reasons:

- Defining a standard and unified model of the collected data and linked it with their contexts. This model will be shareable and exploited between objects as well as between humans.

- Facilitating the discovery, integration, manipulation, and configuration of clinical devices. 
- Supporting reasoning mechanism on the defined context to infer intelligent decision.

For this end, we aim to extend our proposed HealthloT ontology Rhayem, Mhiri, and Gargouri 2017) in order to cover and express diverse contexts that affect the diagnosis and monitoring of both MO's and patients' states missed in the previous version.

Furthermore, to build the present ontology, we relied to some relevant existing one such as SSN Compton et al. 2012. through the ssn prefix, loTCSeydoux et al. 2016), loT-liteBermudez-Edo et al. [2016) with the iot-lite prefix, Time ontology Hobbs and Pan 2006 with the To prefix, Geonamesontology through the geo prefix and MOO ontology Wannous, Malki, Bouju, and Vincent 2013) through the moo prefix.

Formally, we define our ontology as 3-tuples: $\mathrm{O}=(\mathrm{C}, \mathrm{A}, \mathrm{I})$ where :

- C: set of concepts

- A : set of axioms between concepts

- I: set of instances of each concept

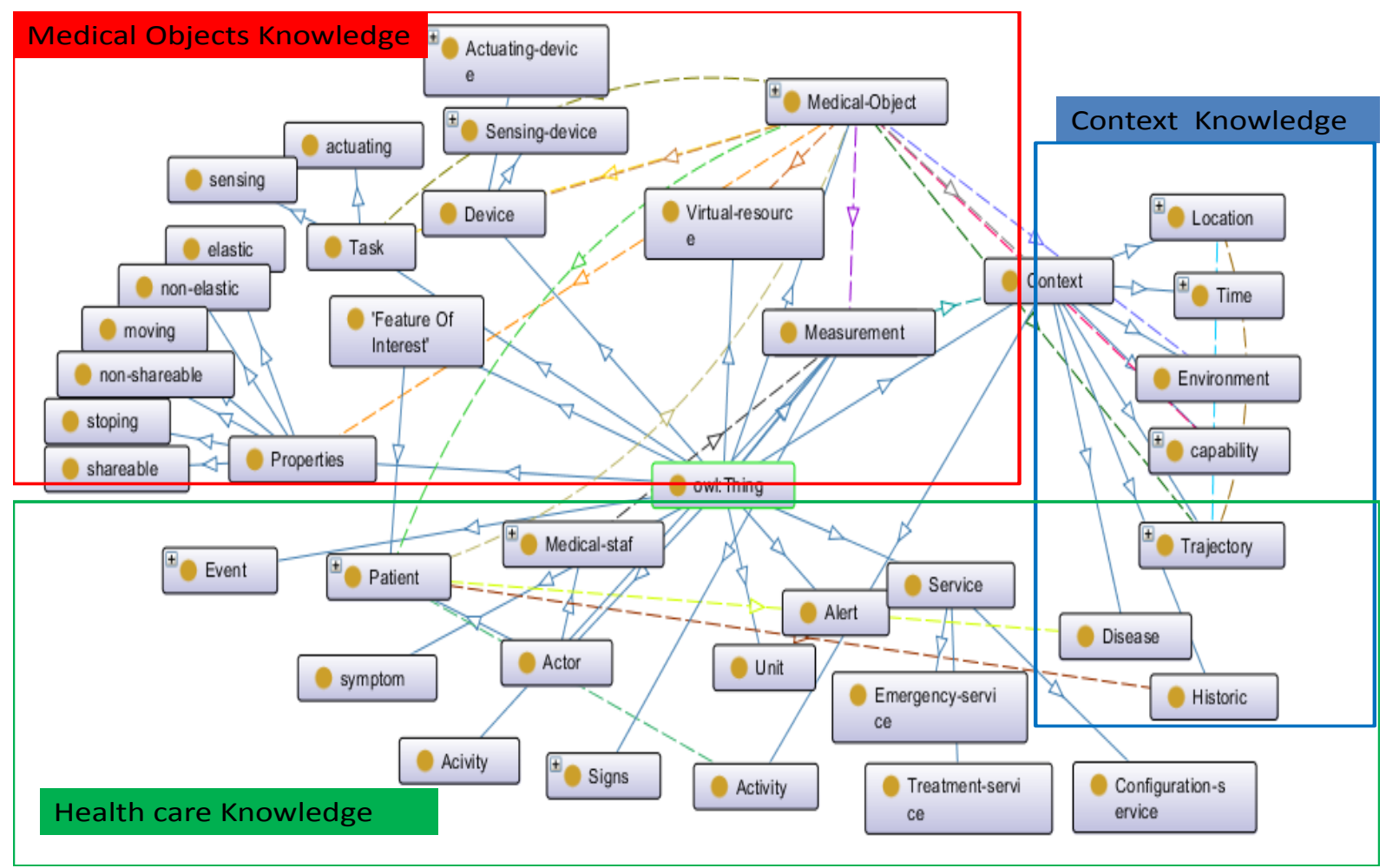

FIGURE 3 HealthloT Ontology

The main concepts of our ontology are classified into three categories: concepts that represent the knowledge about MCO, the knowledge about the patient states and that about their contexts.

\section{1 | MCO Knowledge}

This step is conceived to model the heterogeneous medical connected objects and their specificities. The main defined concepts are as follows: HIoT:Medical-object represents the semantic of heterogeneous medical connected objects used for remote patient monitoring. For example, the blood glucose monitoring device, blood pressure monitoring device, smartphone, and the medical box represent the MCOs used to monitor pregnant woman who has a gestational diabetes disease.

HloT: virtual-resource class is proposed to define one of the main loT goals, which is the virtualization of real-world objects in order to facilitate their management and configuration. 
ssn:Device it is a concept extended from the SSN ontology and has two sub-classes: ssn:Sensing-device and iot-lite:actuating-device. The first represents medical-sensor and RFIDs tags which are responsible for the detection of the occurring event and the second describes the actuators acting on the environment. The sensors used for gestational diabetes monitoring such as the blood glucose sensor, the blood pressure sensor, the ECG sensors, and so one, are presented as instances of the sensing device concept. The vibrator, screen, alarm represent the instances of the actuating device concept.

HIOT:properties represents the properties of the used connected-objects. Thereby, we extend some properties from cloud resource that are satisfied by loT resources such as shareable/non-shareable, elastic/non-elastic, limited and not-limited. Other properties that we can take into consideration in the loT are "moving" and "stop". These properties are modeled as sub-classes of "HloT:IoT-properties" concept. Details about these properties are given in Table 5

TABLE 5 Internet of Things resources properties

\begin{tabular}{|l|l|}
\hline Properties & Definition \\
\hline Shareable & loT resources can perform two task at the same time. \\
\hline non-shareable & loT resources is capable to execute just one task at a given time \\
\hline limited & $\begin{array}{l}\text { In almost of cases loT resources have a maximum capacity as for instance with battery life time and } \\
\text { energy consumption that if it is reached than the loT resource is no longer working }\end{array}$ \\
\hline non limited & loT resources have unlimited capacity \\
\hline elastic & $\begin{array}{l}\text { it's possible to add/remove resources in order to increase their capability (memory, lifetime, response } \\
\text { time, etc) }\end{array}$ \\
\hline non-elastic & there is no possibility to change the capability of loT resources \\
\hline stop & loT resources are deployed in a specific environement during a predefined period. \\
\hline move & loT resources are in moving state if their environment of deployment change during time. \\
\hline
\end{tabular}

HloT:Task: it allows the description of the allocated task by the connected objects and the embedded devices (e.g. sensing blood glucose level, triggering treatment alerts).

\section{2 | Patient Knowledge}

In order to represent the health state of the monitored patient, it is necessary to represent some knowledge from the medical sector. At this level, we adopt the Systematized Nomenclature of Medicine Clinical Terms (SNOMED-CT) 9 standard. These concepts can be briefly described as follows: HIoT:Measurement is designed to represent the semantics of massive quantity of health data obtained from medical connected objects. It has two sub-classes HIoT:signs and HIoT:Activity. The HIoT:signs defines the detected vital signs of the patient such as the blood glucose, the blood pressure, heartbeat, and so on which are modeled as sub-classes of this concept. HloT:Alert contains different categories of alerts that can be generated by the actuator device. These alerts can be for objects management or for patient monitoring (treatment adjsutement, emergency call for ambulance). HIoT:Event represents an abnormal detected event from the MCO. It refers to the healthcare event such as hyperglycemia, hypertension, etc. HIoT:Risk: represents the health complications of uncontrolled disease that may happen.

HIoT:Actor determines the principal actors in the healthcare domain, such as HIoT:Patient, HloT:medical-staff.

HIoT:Service describes various services that can be generated by the medical connected objects. These services can be classified into several categories such as treatment services (HloT:treatment), emergency services when the patient's state is critical (HIoT:emergency-service) and configuration services which contain the state of the connected objects and the proposed solution.

HIoT:Symptom defines the changes in patient behavior and sensations about the disease.

${ }^{9}$ http://bioportal.bioontology.org/ontologies/SNOMEDCT/ 


\section{3 | Context Knowledge}

HІоT:Context characterizes the MCOs and the obtained data about the monitored patient. In fact, the state of both MCOs and the patient's health changes continuously according to several factors. It is fundamental to consider these factors during the configuration of the MCO state and during the diagnosis of the patient's state in order to propose a precise and suitable services.

\subsection{1 | Context related to the medical objects deployment}

- To:Time: describes the medical connected objects' employment time and the temporal validity of their captured data stream. It has several sub-classes extended from the time ontology as for instnace with To:duration, To:instant, To:interval.

- goe:Location: describes the surrounding environment of the employed objects. This concept has two subclasses, including the IndoorLocation and Outdoor-Location in order to express the functioning of the connected objects in a large scale.

- Moo:Trajectory: extended from MOO ontology Wannous et al. 2013. It allows representing the mobility characteristics of the connected objects. Trajectory refers to a list of locations that the object crosses during a predefined period. This concept is related to the Location concept with "has-source" and "has-destination" object properties and to the Time concept with "starts" and "ends" properties.

- Capability: is defined by the HIoT:Capability class. It represents the network context, the resource context, and the sensing and actuating capability context (e.g. energy capability, memory capability, life cycle capability). HIoT:Capability has several sub-classes HloT:SensingCapability, san:Actuating-Capability, HIoT: Tags-capability, HloT:network, and HloT:MO-Capability concepts.

- Environment: is modeled with the HIoT:Environment concept, which determines different factors (humidity, temperature, etc.) that can influence the state of the connected objects and the validity of the detected measurement.

\subsection{2 | Context related to the monitored patient}

To facilitate the management of complex state and provide the suitable treatment, modeling the context relative to the patient is a promising solution. The main proposed classes in our model are described below.

- HloT:Disease: in the health care domain, it is intrinsic to take into account several contexts like the patient disease. For example, in the general case, a person who has a temperature value greater than $37^{\circ} \mathrm{C}$, he has a fever, but for a patient suffering from hypertension disease, he has a fever when the temperature value is greater than $36.5^{\circ} \mathrm{C}$.

- HloT:Historic: represents medical information about patients such as their diseases, their causes, symptoms, historic treatments, and so on. This knowledge helps to provide correct diagnosis, and

- HIOT:Patient: is a primordial context in the healthcare domain. It is a sub-class of "ssn:FeatureofInterset" concept, which refers to the observed and controlled element. This concept defines personal information such as age, sex, weight, etc. that play a primordial role during the diagnosis phase.

- HloT:Activity: defines the patient's activity (e.g. sleeping, running, walking), which are detected by specific sensors such as cameras, accelerometers during health measurements monitoring.

\section{4 | Axioms Modeling}

A set of axioms, between HealthloT ontology concepts, which cover the union, the disjoint and the specialization relations are illustrated in Table 6 Therefore, in order to represent how these concepts are related to each other, we define several semantic relationships, some of them are highlighted in Table 7 .

As example, the Patient class has the relation "has-object" with the Medical-object. Medical-object class is associated with the concept Device via the object property "contains". To define the role of the Medical-Object class, which is the surveillance of a Patient, we propose the object property "monitors" with the range domain ssn:FeatureofInterest and Patient concepts. The objects properties ("has-location" and "has-time") are associated between the Medical-object and both Location and Time concepts respectively. The object property "analysis" is assigned between the Doctor concept and the Measurement concept. 
TABLE 6 HealthloT's axioms

\begin{tabular}{|l|}
\hline Axiom \\
\hline Sensing-device $\subseteq$ Device; Actuating-device $\subseteq$ Device \\
\hline Patient $\subseteq$ Actor; Medica-staff $\subseteq$ Actor; Patient $\subseteq$ ssn: Feature-of-interest \\
\hline context $\equiv$ Time $\sqcup$ Location $\sqcup$ Trajectory $\sqcup$ Capability $\sqcup$ Environment $\sqcup$ Disease $\sqcup$ \\
Patient $\sqcup$ Activity $\sqcup$ Historic \\
\hline Service $\equiv$ Treatment-service $\sqcup$ Emergency-service $\sqcup$ Configuration-service \\
\hline contains $\equiv$ embedded-in \\
\hline OutdoorLocation $\subseteq$ Location ; IndoorLocation $\subseteq$ Location \\
\hline Instant $\equiv$ Time; Interval $\equiv$ Time \\
\hline
\end{tabular}

TABLE 7 HealthloT's objects properties

\begin{tabular}{|l|l|l|}
\hline Object-property & Domain & Range \\
\hline contains & Medical-Object & Device \\
\hline monitors & Medical-Object & Feature-of-interest \\
\hline has-location & Medical-Object & Location \\
\hline detect & Medical-Object & Measurement \\
\hline worn-by & Device & Patient \\
\hline has-object & Patient & Medical-Object \\
\hline has-risk & Patient & Risk \\
\hline allocate & Medical-object & Task \\
\hline has-activity & Patient & Activity \\
\hline analysis & Doctor & Signs \\
\hline propose & Doctor & Treatment \\
\hline has-treatment & Patient & Treatment \\
\hline has-symptom & Patient & Symptom \\
\hline starts & Medical Object & instant \\
\hline finish-at & Medical Object & instant \\
\hline
\end{tabular}

\section{7 | ANALYSIS PHASE}

After the data collection and the semantic knowledge modeling phases, proposing a rule base in order to interpret and exploit this knwoledge is a necessary step. Thereby during this phase, we propose and implement a reasoning process as detailed in Figure 4 It is basically composed of two main steps detailed below.

- Initial diagnosis and data collection step: this phase is carried out by the doctor and the MCOs. Firstly, the doctor diagnoses the patient and recommends the suitable object to be used. Then, the detected measurements of this object are stored in the HealthloT ontology.

- Reasoning step: here, the collected data are analyzed. In fact, several rules are developed based on the SWRL language. These rules consists of an antecedent part specifying the condition that must be met and the consequent part defining the fact that may happen. Our rules are formally defined according to the ECA structure (On Event if Conditions do Actions) Poulovassilis, Papamarkos, and Wood 2006. The event part determines the contexts for triggering the rules. The condition part represents a set of circumstances that should be achieved. The action part specifies the list of actions to be performed if the conditions will be held. The proposed rules are classified into two categories: rules for connected objects' management and others for diagnosis, treatments and notification proposal. These categories will be detailed with regards to the gestational diabetes context. 


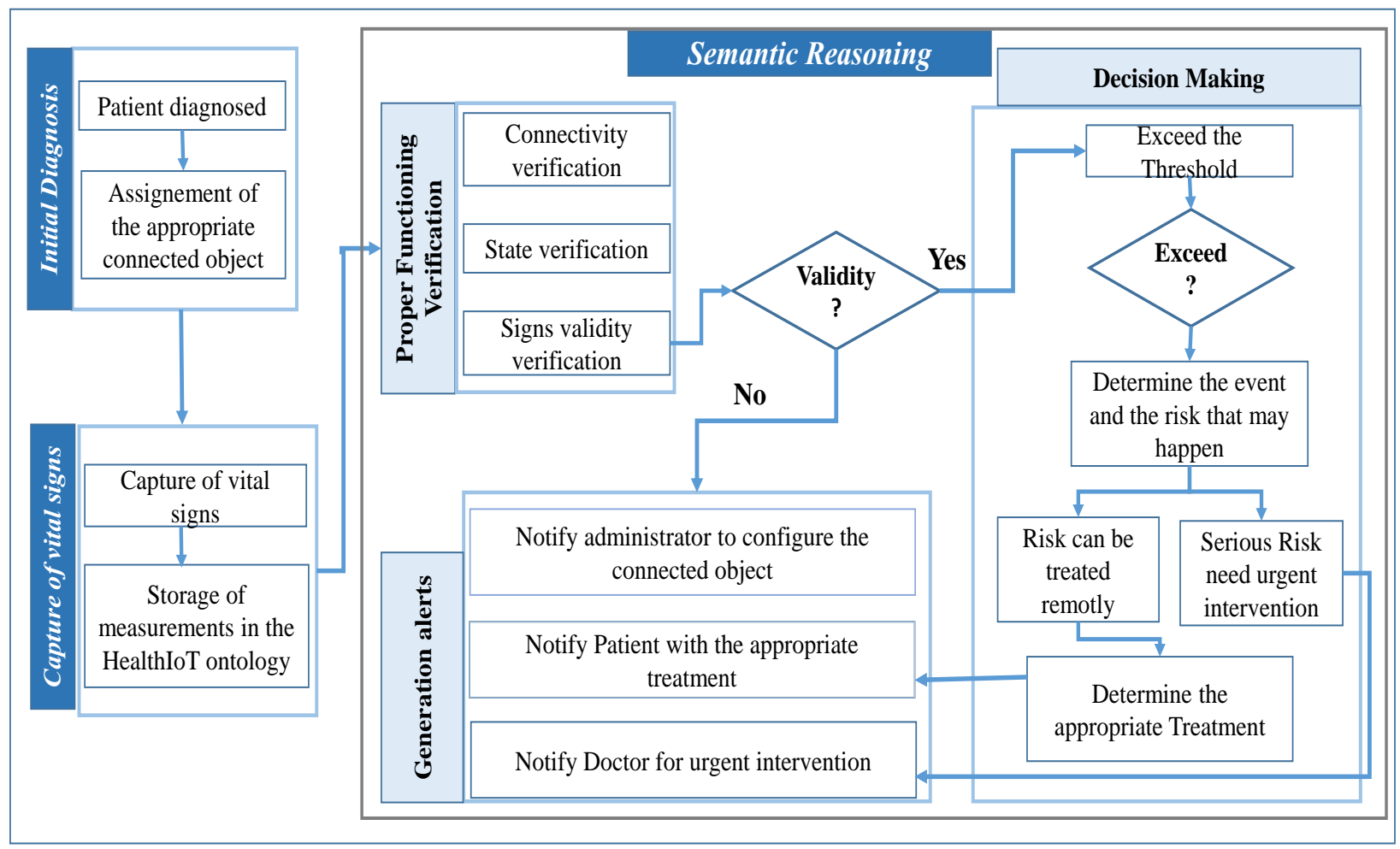

FIGURE 4 Reasoning Process.

\section{1 | Medical Objects Management}

To exploit the MCOs in a reliable and effective way, it is necessary to ensure and check their proper functioning, their adaptability level with diverse contexts and their ability to perform configurations.

In this section, we develop diverse rules some of them are described below, which mainly focus on: i) the diagnosis of the objects state ii) the allocation/de-allocation of tasks, and iii) the verification of their capabilities. We present in ?? some SWRL rules for MCOs management in accordance with the proposed use case.

Determine the mode of MCOs: Connected objects can have three different modes:

- Active mode, which verifies that the object is currently active and it is not available to be, used for other tasks.

- Standby mode: verifies that the object finalizes its work and it becomes available for other tasks;

- Passive mode: indicates that the state of the sensor is out of order and it is not available for new tasks that implies to be replaced by another.

These states should be verified using the time context. For example, the standby mode verification consists of:

Event: A MCO contains a sensing device with a blood glucose type and used by a pregnant woman has finished its task and waited for another one. (the instant when the sensor accomplishes its task should be before the actual time). This event was triggered thanks to the temporal built-ins ("temporal:before" and the subject "now") used from the temporal ontology.

Condition: The lifetime of the sensing device should be after the actual time. For that, we use the temporal built-ins "temporal:after".

Action: The medical object becomes in standby mode. 


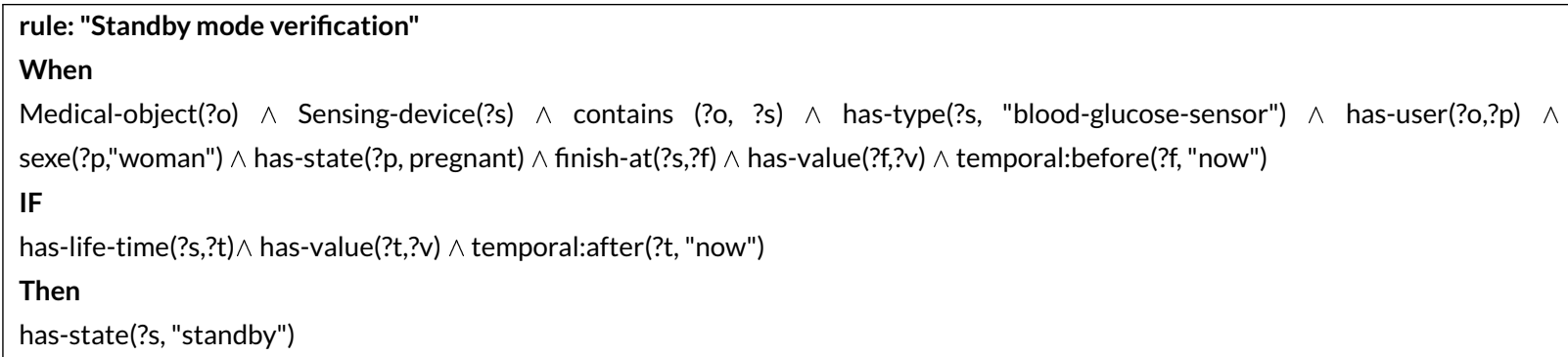

Verify the availability of MCOs: In this category, the proposed rules determine if the MCOs are available for new tasks or not. These rules depend on the result of the verification of the mode rules. If the MCO was in active or passive state, it is not available. However, if it is in a standby mode, it becomes available to execute other tasks. The proposed example consists of:

Event: a MCO that contains a sensing device with a blood glucose type is used by a pregnant woman.

Condition: the $\mathrm{MCO}$ is in a standby mode.

Action: the MCO is available to execute a new task (e.g. the detection of the blood glucose level after two hours).

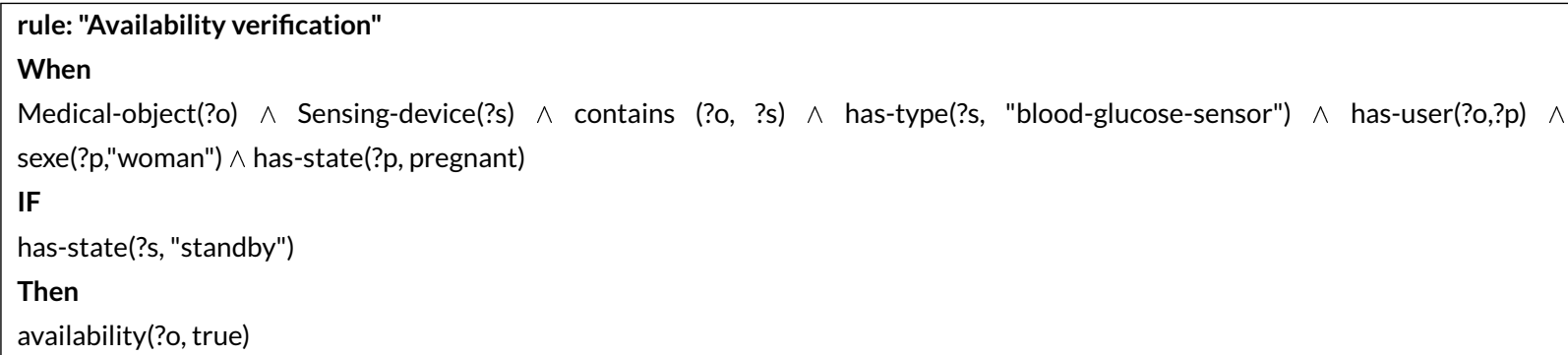

Task allocation: The main goal of these rules is to allocate/de-allocate tasks for MCOs. They are based on the verification of the mode and the availability rules. Therefore, if the MCOs is available, it can allocate a new task. For example, the suggested rule in this paper is formed by: Event: a MCO contains a sensing device with a blood glucose type turns passive (e.g. lifetime exceeded).

Condition: another MCO contains a sensing device with the same type of the previous $\mathrm{MCO}$ is available to execute a new task.

Action: the task of the first MCO is allocated by the second one that will be attached to the monitored patient.

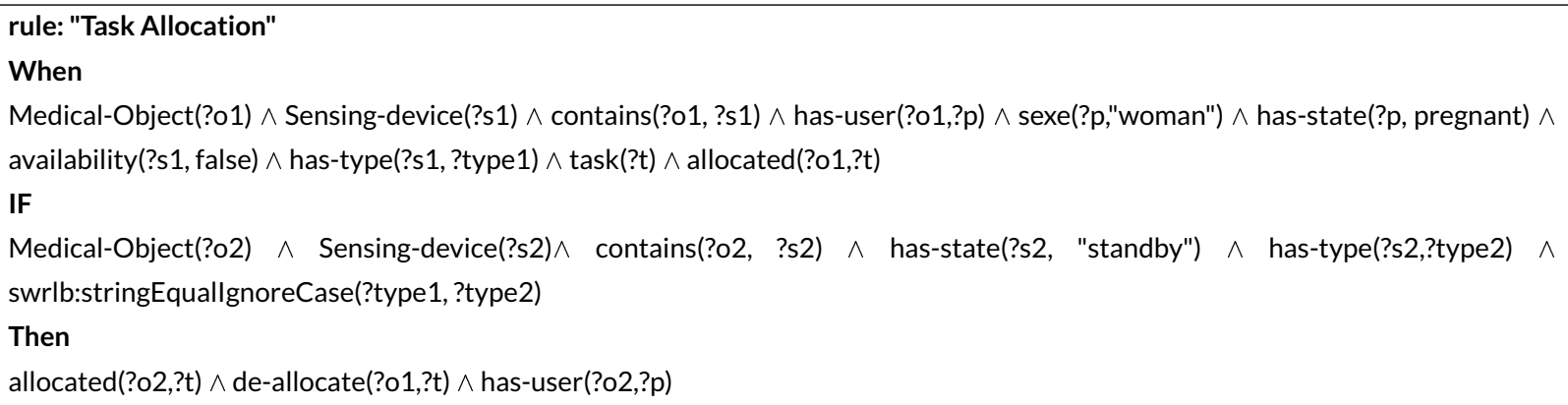

Capability Verification: To examine the proper functioning and capabilities (battery level, RAM memory, energy consumption, etc) of the medical objects, it is very important to propose various rules. The following rule consists of:

Event: MCO executes a task

Condition: its battery level is $10 \%$

Action: the state of the battery is Low and the mode of its sensing-device becomes standby (sleep state) to save energy consumption. 


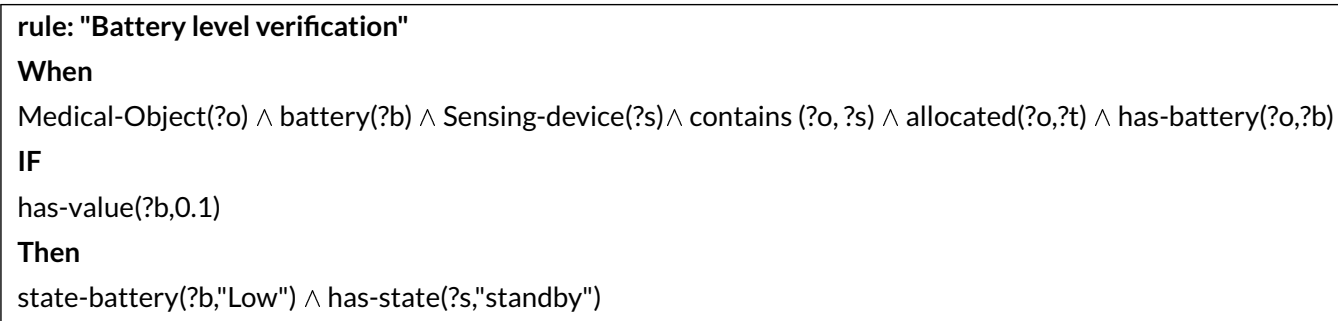

Mobility Verification: MCOs can be either in fixed or moving states. In fact, moving state defines their dynamic position that changes continuously instead of the fixed one that designates their constant location. Verifying this state needs spatio-temporal data. Therefore, based on the temporal:duration builtins, and the Time, Location and Trajectory contexts, we proposed an SWRL rule that determines if the location of this object changes. We have considered a time interval depending on the estimated period needed for the patient monitoring task that takes into account the patient's state and the complexity of the disease. For example, we supposed that a pregnant woman needs to monitor her blood glucose 8 times per day, separated by two hours. In these times, we aim to control the mobility of the MCO. Accordingly, it will be easier to allocate tasks in case of failure of MCO by considering only the nearest objects. In addition, it helps to control the activity of the patient. It consists of:

Event: MCO has a trajectory that is composed of a set of crossed locations during an interval of time

Condition: the location is the same during two hours.

Action: $\mathrm{MCO}$ object is in stopping state and consequently the patient is motionless.

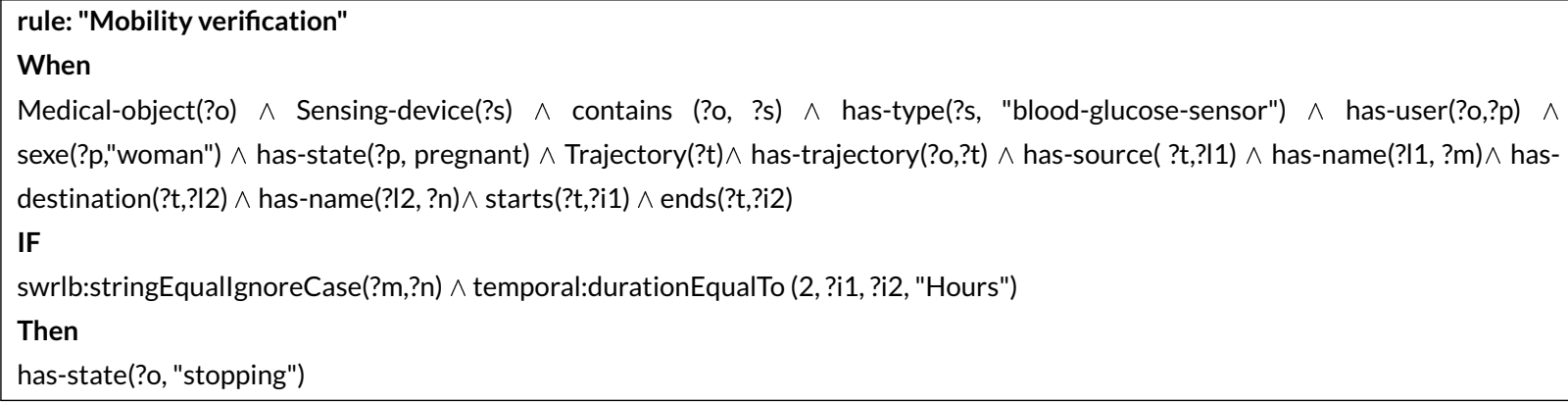

\section{2 | Patient State Diagnosis and Decision Making}

One of the main contributions of this work is to propose a decision-making process namely Measure-Event-Risk-Treatment-Alert (M-E-R-T-A) that takes into account the whole remote diagnosis process for the patient. M-E-R-T-A starts with the analysis of the obtained measures, then the detection of the health event that may happen, the prediction of risk complication, and finishes with the proposition of the adequate treatment and the notification of the patient. Figure 5s shows an example that allows interpreting the detected data with a Glycemia sensor based on M-E-R-T-A. Thereby, the analysis of glycemia level leads to the detection of hyperglycemia event and the possible risk that may happen as the fetal loss for the pregnant woman. After that, the next phase determines and notifies the patient with the appropriate treatment (Metformin Mylan).

To perform this process, we develop diverse SWRL rules to treat five different health measurements (temperature, blood pressure, heart rate, blood glucose, and cholesterol). In the next sub-sections, we describe some rules in accordance to the proposed use case (gestational diabetes context).

Validity of Data; For the purpose to analyze the obtained data in real-time, we define the following to verify the validity of the blood glucose. It consists of:

Event: MCO detects blood glucose level of a pregnant woman.

Condition: the deadline of the obtained blood glucose is greater than the time required for data access and analysis by the doctor. To verify this condition we exploit the temporal SWRL builtins. temporal:before and the subject "now" that refers to the actual time.

Action: The blood glucose is valid to be processed and analyzed. 


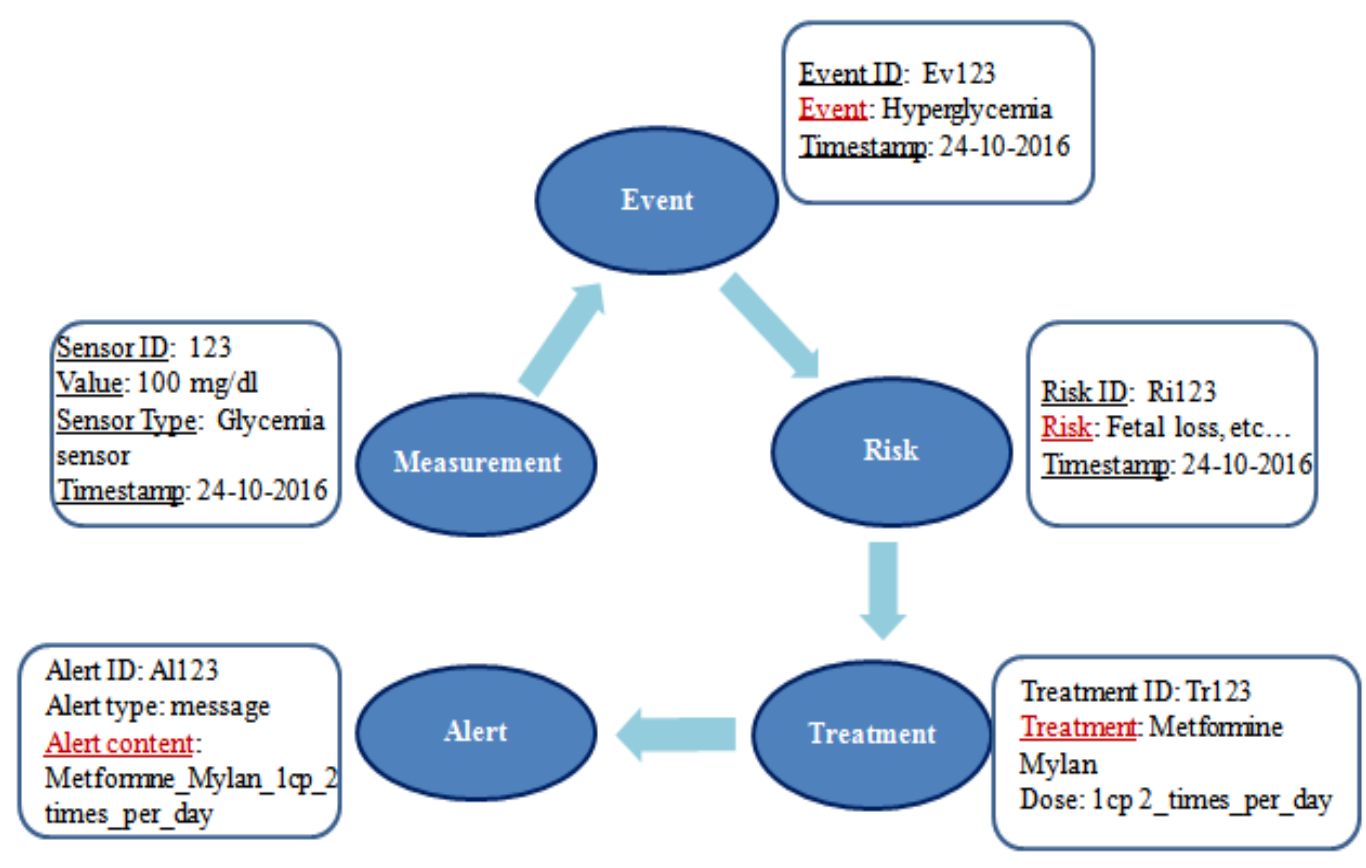

FIGURE 5 M-E-R-T-A: diagnostic and decision making method

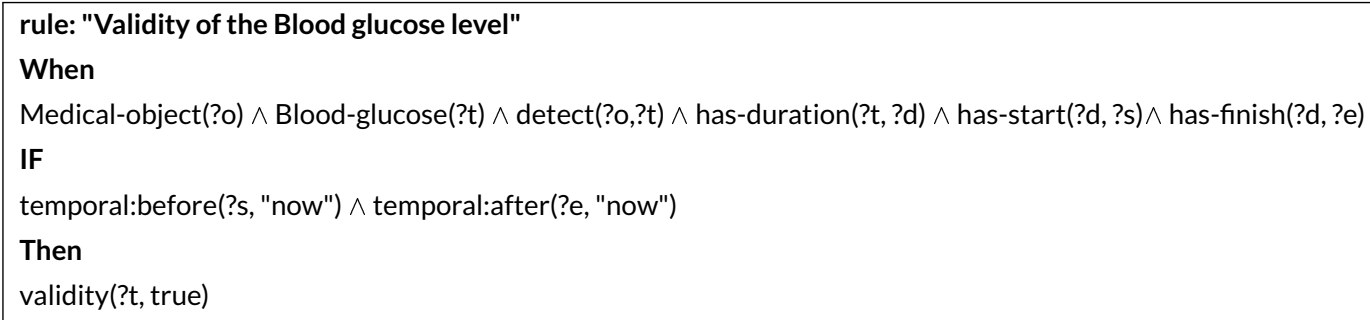

Events detection; These rules are proposed to enable medical staff to predict and detect events on time. For this end, we define several SWRL rules which aim to examine the patients' vital signs from different contexts in real time and to provide an adequate solution. For example, the next rule identifies the hyperglycemia event (Gestational diabetes) for a pregnant woman. Therefore, in the normal case, hyperglycemia is diagnosed when the blood glucose level is greater than or equal to $126 \mathrm{mg} / \mathrm{dl}$. But, in the case of pregnant woman, it is diagnosed if it is greater than or equal to $92 \mathrm{mg} / \mathrm{dl}$. This rule consists of:

Event: MCO detects blood glucose level of a pregnant woman.

Condition: The detected blood glucose is valid and it is greater than $92 \mathrm{mg} / \mathrm{dl}$ and the pregnancy is meanwhile the forth and ninth months. We use the temporal built-ins "temporal:durationGreaterThan" and "temporal:durationLessThan" to analyze the pregnancy duration of the patient. Action: Hyperglycemia event is detected.

\section{rule: "Hyperglycemia event detection"}

When

Patient(?p)^ sexe(?p, "woman")^ has-state(?p, pregnant) $\wedge$ start-at(pregnant,?s) $\wedge$ temporal:durationGreaterThan (4, ?s, "now", "Months") $\wedge$ temporal:durationLessThan (9, ?s, "now", "Months")^ Medical-object(?o) $\wedge$ has-user(?o,?p) $\wedge$ Blood-glucose(?t) $\wedge$ detect(?o,?t)

IF

validity(?t, true) $\wedge$ has-value(?t,?v) $\wedge$ swrlb:greaterThan $(? v, 92) \wedge$ has-unit(?t, ?u) $\wedge$ has-name $($ ?u, "mg/dl")

Then

has-event(?p, Hyperglycemia) $\wedge$ has-disease(?p, Gestational-diabete) 
Risk anticipation; Risk prediction and management are very paramount in the healthcare domain. Thereby, the main goal in this phase is to control and detect potential health risks in order to prevent it and minimize critical crises. For this reason, it is very important to propose a risk management plan that should identify the controlled situation, the critical effect of the result if it happens and its impact.

According to the $\mathrm{WHO}$, uncontrolled diabetes during pregnancy give rise to a very dangerous effect on both mother and child as for instance with the risk of fetal loss, congenital malformations, stillbirth, and obstetric complications. However, the degree of severity of these risks depends on other factors and symptom such as hypertension, cramping, age greater than 40 years and so on. The next rule is formed as:

Event: an hyperglycemia is detected in a pregnant woman.

Condition: the woman has another disease (hypertension), her age is greater than or equal to 40 , she has a cramping symptom. Action: This woman has a high risk of fetal loss.

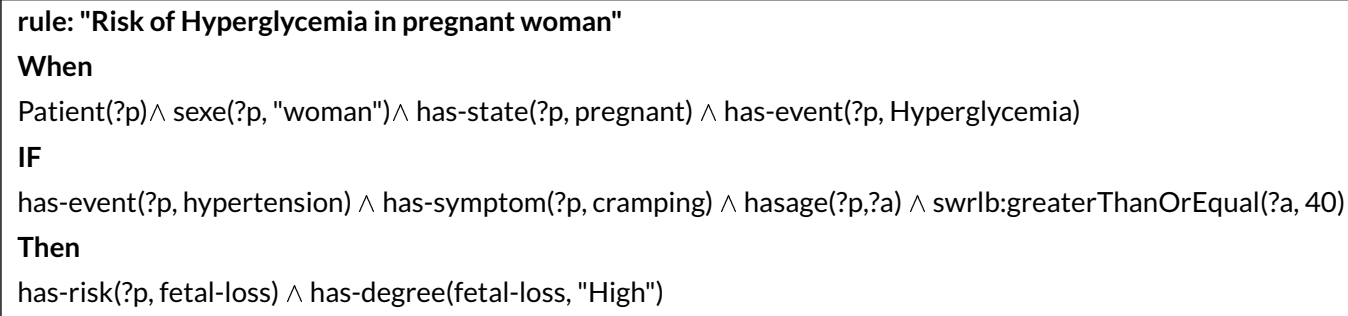

Treatment proposition; In this phase, diverse rules are defined to assist doctors in making an efficient and suitable treatment according to the diagnosis results. The proposed treatment can be a lifestyle recommendation, drugs proposition, and adjustment, or emergency service that requires the quick intervention of the medical staff. The next rule describes an emergency service for a pregnant woman. This rule is defined as Event: an hyperglycemia is detected in a pregnant woman.

Condition: the woman has a high risk of fetal loss.

Action: the woman needs an emergency service(e.g. ambulance).

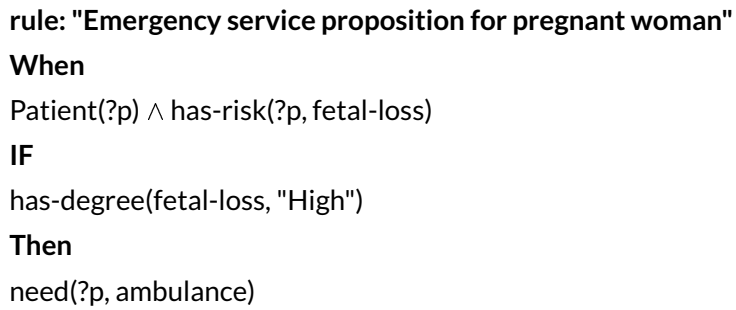

Rules for alert generation; A generated alert from the MCOs is classified into three categories according to their importance (normal, medium and urgent). The normal alert is automatically produced by the MCOs where the analysis of the data is performed by the controller devices, and the decision is provided within the actuator devices. This kind of alert is usually used in simple cases (e.g., message contains the value of the detected signs). The medium alert is proposed in cases that need the intervention of medical staff, the data would be transferred to the healthcare professionals to analyze it and to propose the adequate alert that would then be sent to the MCOs of the patient. The urgent alert is provided when the medical staff detects a critical case, which needs urgent intervention. The following rule represents an example of a normal alert, which consists of: Event: hyperglycemia is detected in a pregnant woman.

Condition: The woman has a MCO that contains an alarm actuator.

Action: The alarm state will be "ON" and the received message was "High blood glucose, contact your doctor".

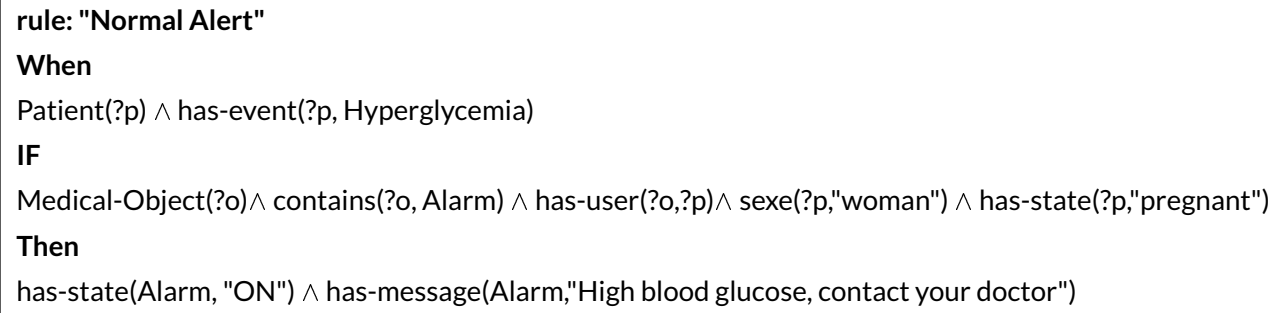

The next one describes an emergency alert triggered in critical cases. It is formed as

Event: the patient (pregnant woman) needs emergency service (ambulance).

Condition: a hospital has an available ambulance. 
Action: The actuator of the ambulance is on state "ON" and has a message, which indicates the name and the location of the corresponding patient.

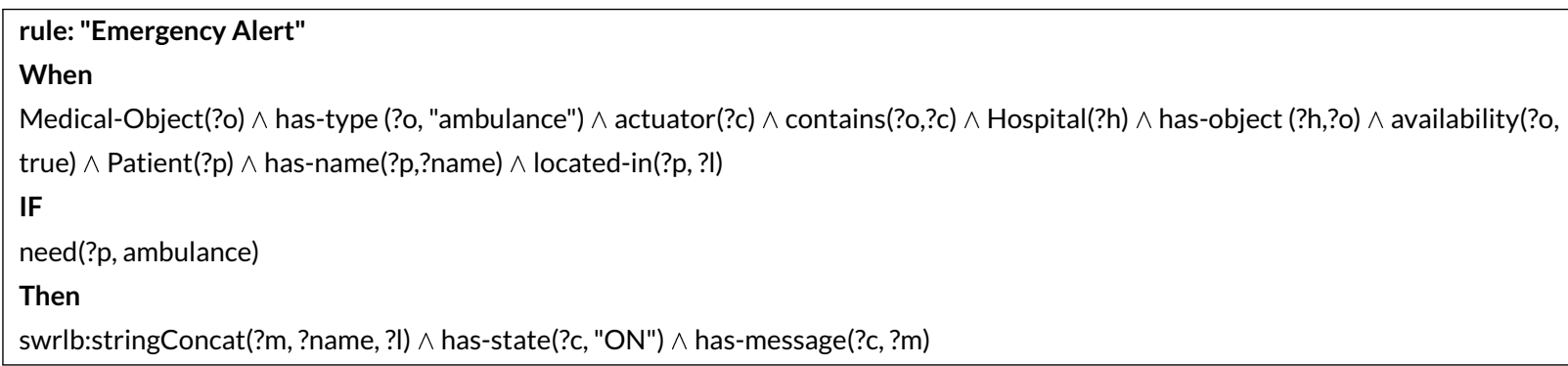

\section{I IMPLEMENTATION PHASE}

Once our Knowledge-base is defined referring to previous phases, it is necessary to be exploited by end-users. From this regard, the main goal of this implementation phase is to develop an loT-based clinical decision support system (IoT Medicare system). This system is integrated with query and inference engine (Drools engine) developed with SWRLAPI, OWLAPI, and Jena APIs which are used to deal with SWRL rules, and SPARQL queries. To provide a suitable decision, this system takes into account the health data that describes the patient's information and technical data that represents the state of the medical objects. The loT Medicare system consists mainly of three modules: Medical Objects Management, Diagnosis and treatment module, and a Notification module. The connected object management module deals with ensuring the proper functioning of the connected objects. The second module focuses on the analysis and the interpretation of the detected patient vital signs, to treat and prevent diseases. The notification module allows notifying patients with the decision of the doctor.

The reliable exploitation of the loT system requires a secure actor authentication to protect the accessed information through a password stored in the HealthloT ontology. After that, the end user is able to ask simply a query using easy ways (button choose, or menu choose). This query is transferred to the knowledge base to run an inference engine (Drools engine). The latter, firstly, loads the ontology on the basis of OWLAPI and executes the developed SWRL rules. Subsequently, it gives the actors the appropriate decision through the obtained inferred results.

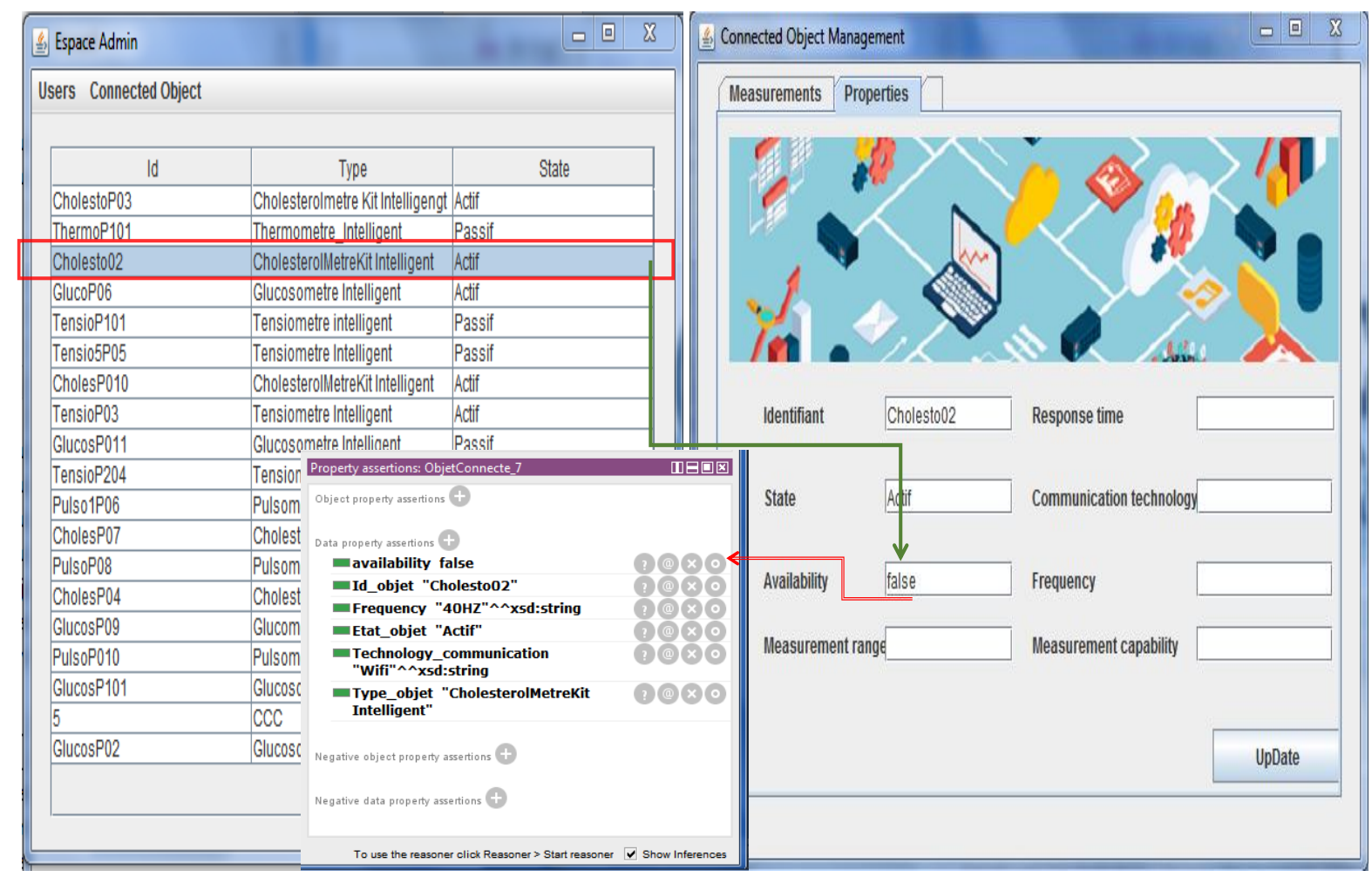

FIGURE 6 Update MO's state 


\section{1 | MCO Management Module}

With the continuous growth of medical connected objects, a manual monitoring of their states has become infeasible and very hard. Therefore, it is paramount to propose a monitoring system that enables the administrator user to:

- Facilitate the search, the update and the exploitation of these devices.

- Ensure a periodic monitoring of medical connected objects.

- Help to anticipate problems and provide preventive and predictive maintenance.

The administrator is the responsible for this task. Thus, he is able to monitor the state of the medical object and configure it, add new objects, consult the list of objects, update its properties, among others.

Figure 6 shows an example of updating some parameters of a selected object from the returned list. This list is the result of the SPARQL query illustrated in Figure 7d. This query returns the objects that need a configuration (e.g. object with active mode and with availability true). The administrator can easily change the value of the availability state to false through a user-friendly interface. The confirmation of this task is insured via the "UpDate" button, which executes a SPARQL update query Figure 7a. This query consists of two main operations. The Delete operation removes the "true" value of the data property availability and the insert operation inserts the "false" value.

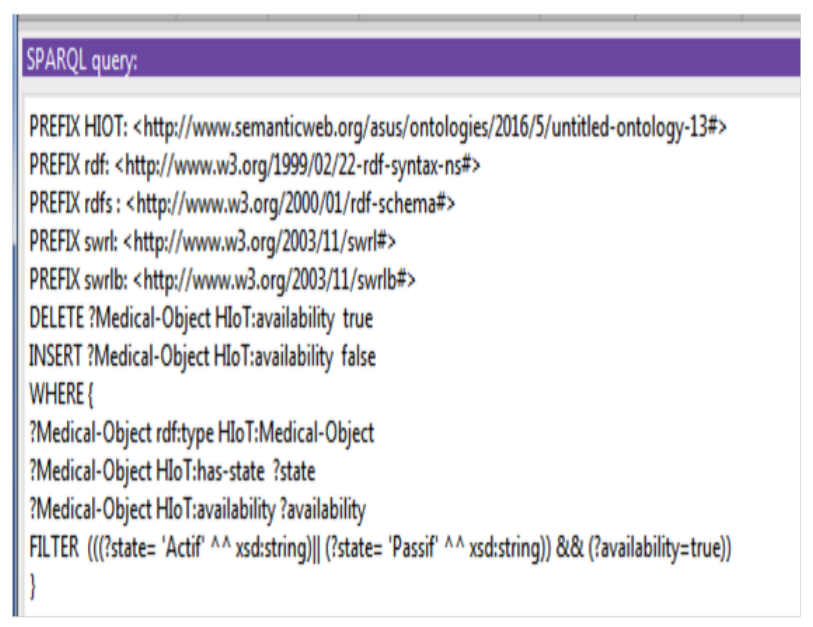

(a) SPARQL Query to update the state of MO.

\begin{tabular}{|c|}
\hline SPARQL query: \\
\hline 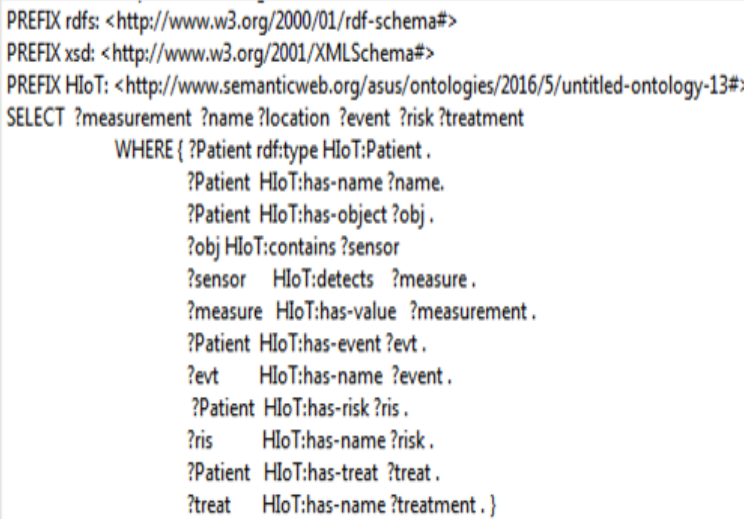 \\
\hline
\end{tabular}

(c) SPARQL Query to select the list of patients, their measurements, the detected events, risks and the proposed treatments.

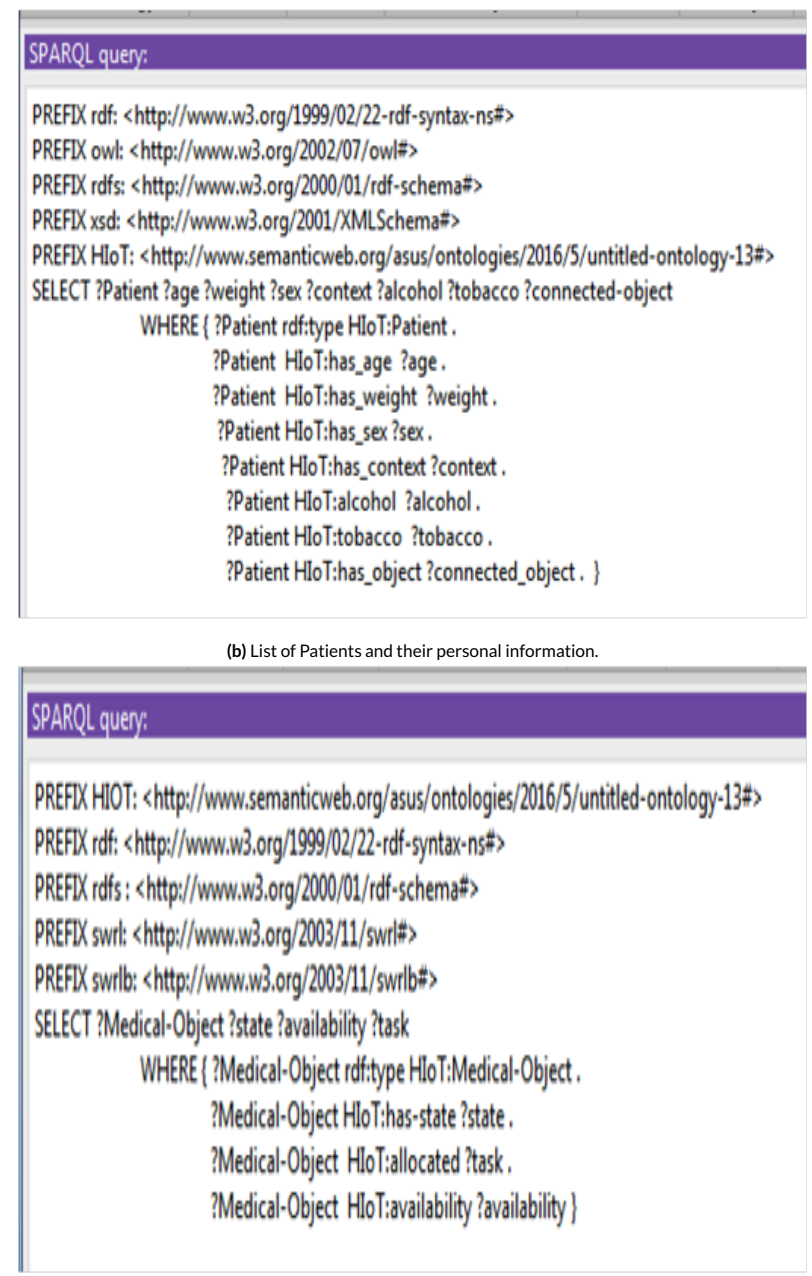

(d) SPARQL Query to select the list MO, their states, their tasks, and their availability. 


\section{2 | Patient State Diagnosis Module}

This module has been designed to describe how healthcare professionals can remotely provide diverse systematic care services including the analysis and diagnosis of the patients' states.

The meaningful interpretation of vital signs obtained from several connected objects; this implies to provide adequate treatments for patients, which presents the main goal of this module. In fact, through a simple authentication, the doctor can perform various functionalities. Firstly, he should specify the appropriate object to be used to monitor the patient's state. Once this object is activated and based on its detected vital signs, he is able to diagnose the patient's state. Then, he can consult the list of patients in charge in order to know their status, their disease record, and followed treatments, notify them with a suitable cure, and so on. When the doctor selects the patient to be monitored, a decision-making interface is displayed in Figure 8 This interface illustrates the obtained vital signs from the patients' medical objects. Accordingly, by clicking on the button "Diagnostic", the doctor can check the patient's actual state, the possible risk and the appropriate treatment for this predicted event. In this interface, the diagnostic, the risk and the treatment areas are alterable in order to offer the doctor the possibility of updating them because the role of a doctor is irreplaceable. After that, through the button "Notify Patient" the doctor sends his decision to the patient.

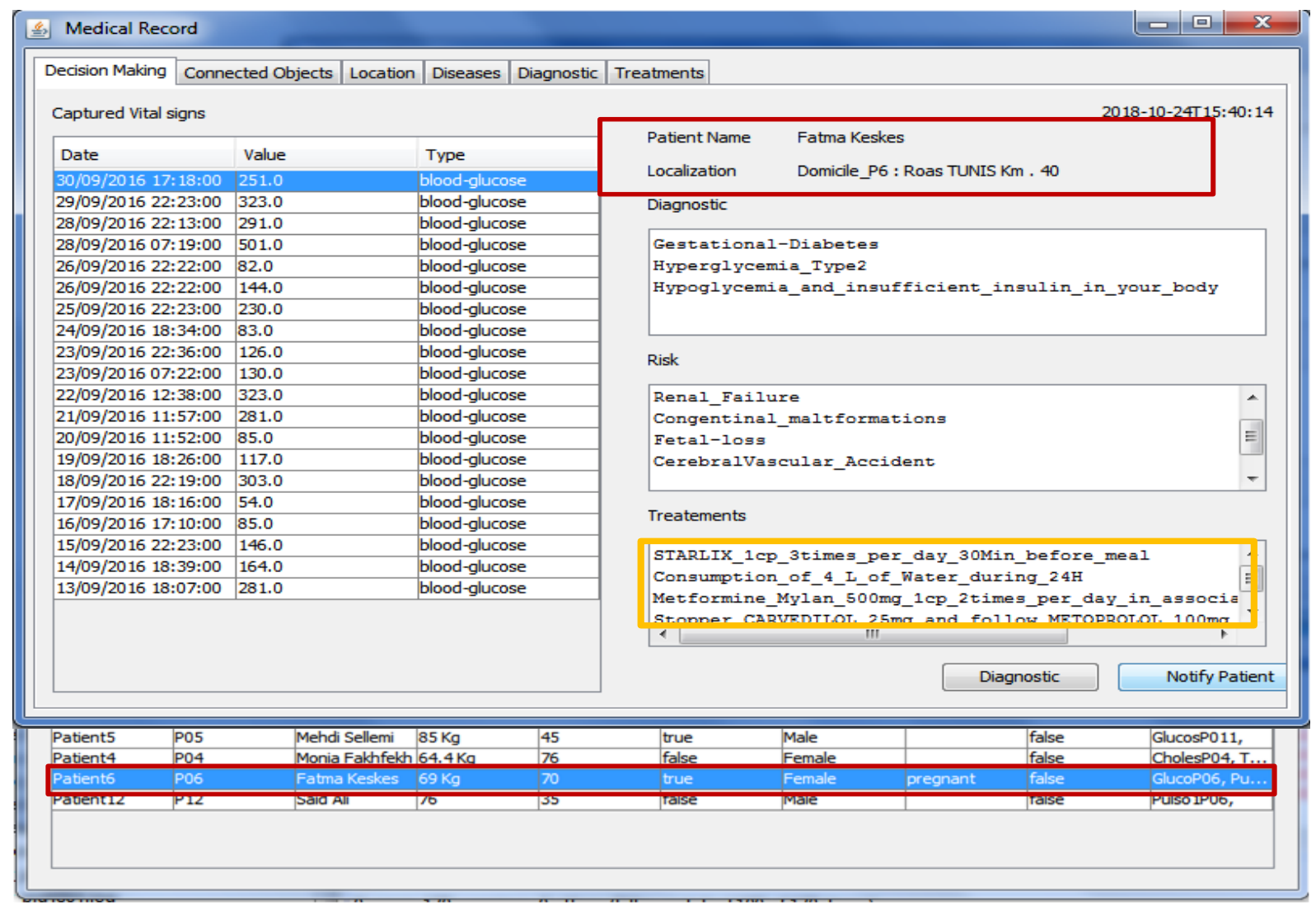

FIGURE 8 Decision Making

\section{3 | Notification Module}

The main reason to design this layer is to make patients capable to receive recommendations and treatments from their doctors and contact them when they face adverse effects following a proposed medication.

This module is very important because it ensures a high health care service delivery during a reasonable time.

Figure 9 represents an example of a notification that contains adequate treatment after the analysis of the state of the selected patient (colored in red) in the last module and how this notification is saved in the knowledge base "HealthloT-Ontology". This module is composed of a diverse menu. The profile menu provides general information about the patient and the suggested treatments by the doctor. The diagnostic menu shows the 
diagnosis events and possible healthcare risks. The measurements menu shows the vital sign's value and the detection time. The treatments menu contains previous treatment taken by the patient. The physicians menu contains general information of the patient's doctor and his web site.

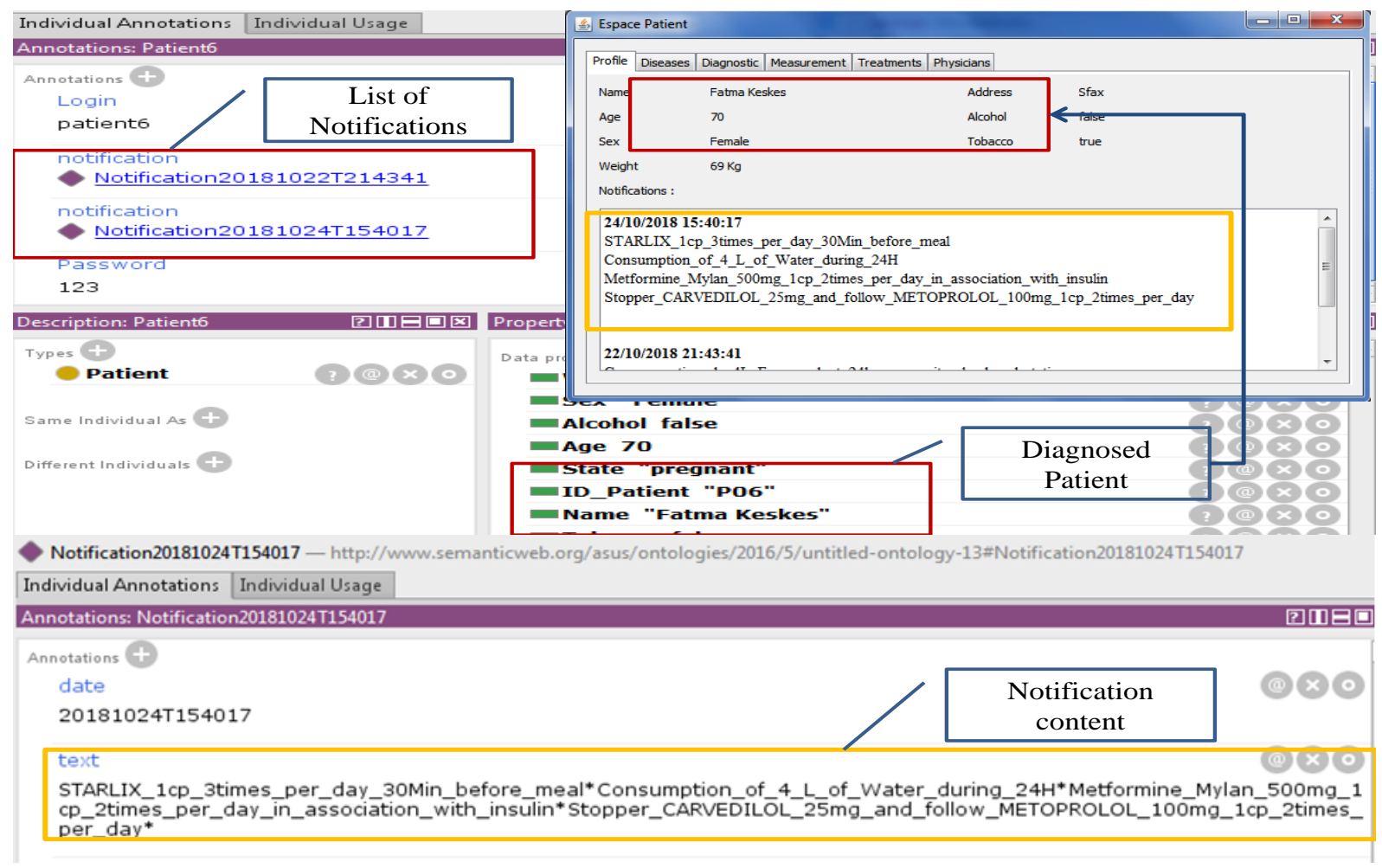

FIGURE 9 Saved Notification in HealthloT-O

\section{9 | EVALUATION}

We evaluate our approach on the functional and technical level. In the technical level, we focus on evaluating the semantic quality of the proposed model (HealthloT-O). In the functional level, we focus on the reasoning performance and the response time of our proposed system "loT Medicare system".

\section{1 | Technical Evaluation: OQUARE Framework}

In order to evaluate the quality of our ontology, we applied the OQuaRE evaluation framework Duque-Ramos et al. 2013, which is mainly based on the Software product quality standards ISO/IEC 25012:2008 (SQuaRE) ISO/IEC] 2008]. In this work, the authors used the SQuaRE characteristics namely structural, functional adequacy, adaptability, maintainability, operability, reliability, and transferability. Each characteristic was assessed with various sub-characteristics and metrics.

Table 8recapitulates these characteristics, their definitions, their sub-characteristics, and the related metrics.

For more details about the relative metrics of each sub-characteristic, how each metric is calculated and how the score is assigned, readers may refer to these works Duque-Ramos et al. 2013, Duque-Ramos, Fernández-Breis, Stevens, Aussenac-Gilles, et al. (2011), Duque-Ramos et al. (2014). Based on these works, our experimentation process is summarized as follows:

1. Firstly, we calculated the value of each metric for each sub-characteristic (note that one metric can be used for several sub-characteristics). 
TABLE 8 OQuaRE Charecteristics and metrics

\begin{tabular}{|c|c|c|c|}
\hline Characteristics & Definitions & Sub-characteristics & Metrics \\
\hline Structural & $\begin{array}{l}\text { Allows evaluating the ontology based on diverse } \\
\text { formal and semantic ontological properties }\end{array}$ & $\begin{array}{l}\text { Formal, } \quad \text { Cohe- } \\
\text { sion, } \quad \text { Redundancy } \\
\text { Tangledness }\end{array}$ & $\begin{array}{l}\text { RROnto, LCOMOnto, } \\
\text { TMOnto, ANOnto }\end{array}$ \\
\hline $\begin{array}{l}\text { Functional Ade- } \\
\text { quacy }\end{array}$ & $\begin{array}{l}\text { Evaluates the degree of the ontology to execute } \\
\text { concrete function purposes }\end{array}$ & $\begin{array}{l}\text { Controlled vocabu- } \\
\text { lary, Consistent search } \\
\text { and query, knowledge } \\
\text { acquisition, results rep- } \\
\text { resentation, knowledge } \\
\text { reuse }\end{array}$ & $\begin{array}{l}\text { ANOnto, RROnto, AROnto, } \\
\text { INROnto, } \\
\text { NOMOnto, }\end{array}$ \\
\hline Reliability & $\begin{array}{l}\text { Determines the degree of the ontology to main- } \\
\text { tain the performance level under several condi- } \\
\text { tions }\end{array}$ & $\begin{array}{l}\text { Availability, } \\
\text { Recoverability }\end{array}$ & $\begin{array}{l}\text { WMCOnto, DITOnto, } \\
\text { NOMOnto, LCOMOnto }\end{array}$ \\
\hline Maintenability & $\begin{array}{l}\text { Determines the flexibility of the developed model } \\
\text { to adapt to changes in the environment, require- } \\
\text { ments, and functional specification }\end{array}$ & $\begin{array}{l}\text { Modularity, Reusability, } \\
\text { Analysability, Change- } \\
\text { ability, Modification } \\
\text { Stability, Testability }\end{array}$ & $\begin{array}{l}\text { WMCOnto, } \\
\text { NOCOnto, } \\
\text { NOMOnto, } \\
\text { CBOOnto }\end{array}$ \\
\hline Compatibility & $\begin{array}{l}\text { Checks how much the ontology can be deployed } \\
\text { for different applications and with different soft- } \\
\text { ware }\end{array}$ & $\begin{array}{l}\text { Replaceability, } \\
\text { Adaptability }\end{array}$ & $\begin{array}{l}\text { WMCOnto, } \\
\text { RFCOnto, } \\
\text { CBOOnto }\end{array}$ \\
\hline Operability & $\begin{array}{l}\text { Verifies how much the ontology enables users to } \\
\text { learn its application }\end{array}$ & Learnability & $\begin{array}{l}\text { WMCOnto, } \\
\text { NOMOnto, } \\
\text { NOCOnto }\end{array}$ \\
\hline
\end{tabular}

2. Secondly, we assigned a score for each metric that shows the acceptability degree of each measurement. The score range varies between 1 and 5. Where 1 means highly unacceptable, 2 unacceptable and improvement is required, 3 minimally acceptable, 4 acceptable, and 5 exceeds the requirements. The mapping process is well detailed in Duque-Ramos et al. 2011.

3. Then, we calculated the average score of these sub-characteristics that is equal to the mean score of all their associated metrics.

4. Finally, we calculated the average score of each characteristic which is equal to the Mean Score of their sub-characteristics.

Figure 10 depicts the score of each quality characteristics of the HealthloT ontology. The structural, functional, reliability as well as the operability characteristics are above average, which reflects the good quality of the HealthloT ontology. However, for the Maintainability and compatibility characteristics, the average score is above 3 (minimally acceptable) but still less than 4. For these characteristics, some metrics should be improved to reach this score. For example, in the maintainability characteristics, LCOMOnto and DITOnto metrics have the score 3 , which is minimally acceptable. Consequently, the HealthloT ontology needs some improvements related to these indicators.

\section{2 | Functional Evaluation}

In this step, we evaluated the reasoning performance of HealthloT ontology and the effect of the quantity of data obtained from medical devices and their contexts on the response time of the loT Medicare system.

\subsection{1 | Reasoning Performance of HealthloT}

To evaluate the effectiveness degree of our model reasoning, three evaluation measures including Recall, Precision, F-Measure were considered using the following equations. We relied on the contingency table as described in Table 9 We denote TP, FP, TN and FN as True Positive (correctly diagnosed instances as required), False Positive (incorrectly diagnosed instances as required), False Negative (incorrectly diagnosed instances as not required), and the True Negative (correctly diagnosed instances as not required). 


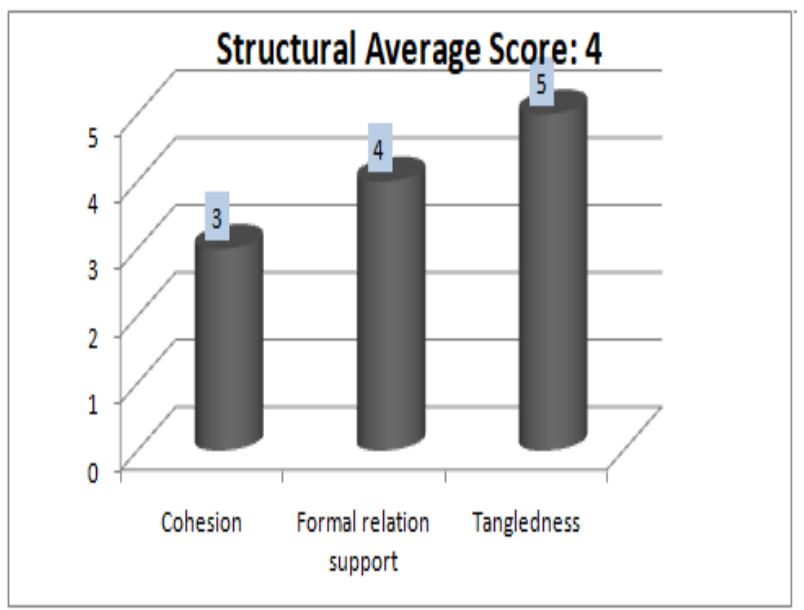

(a)

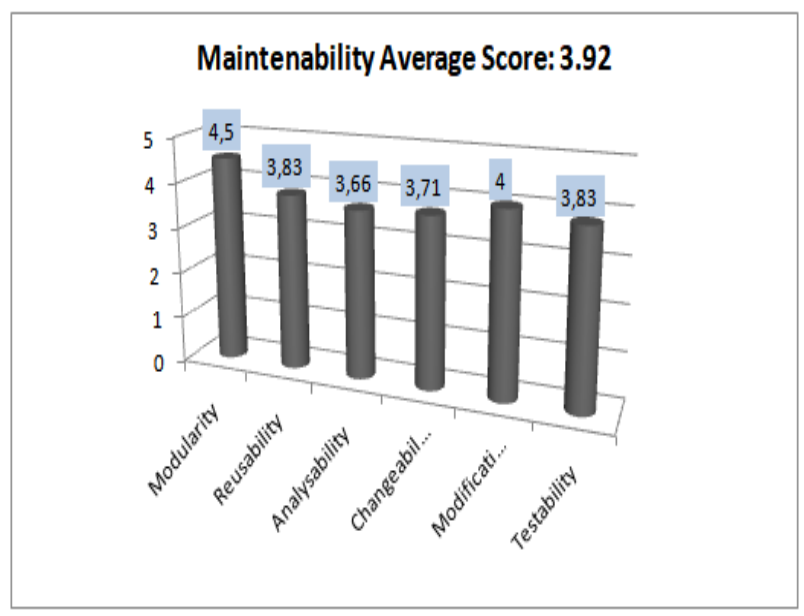

(c)

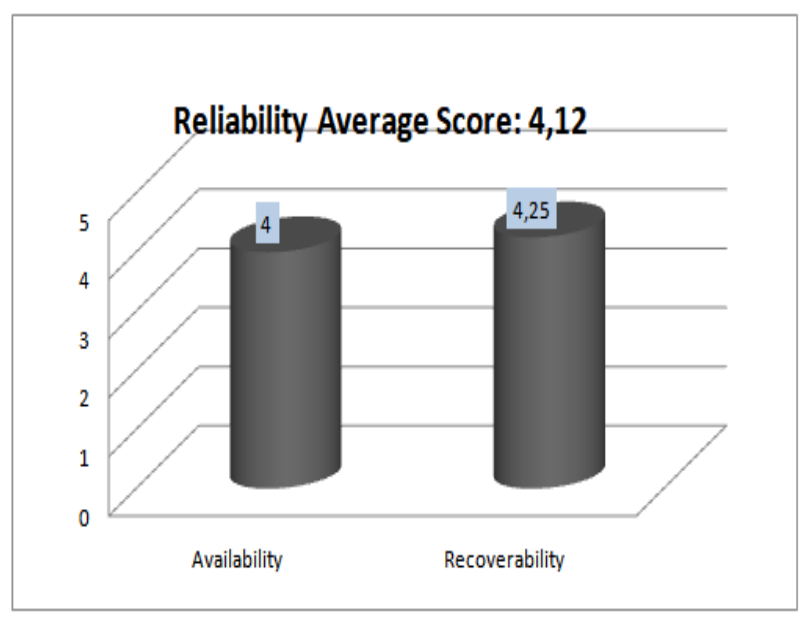

(e)
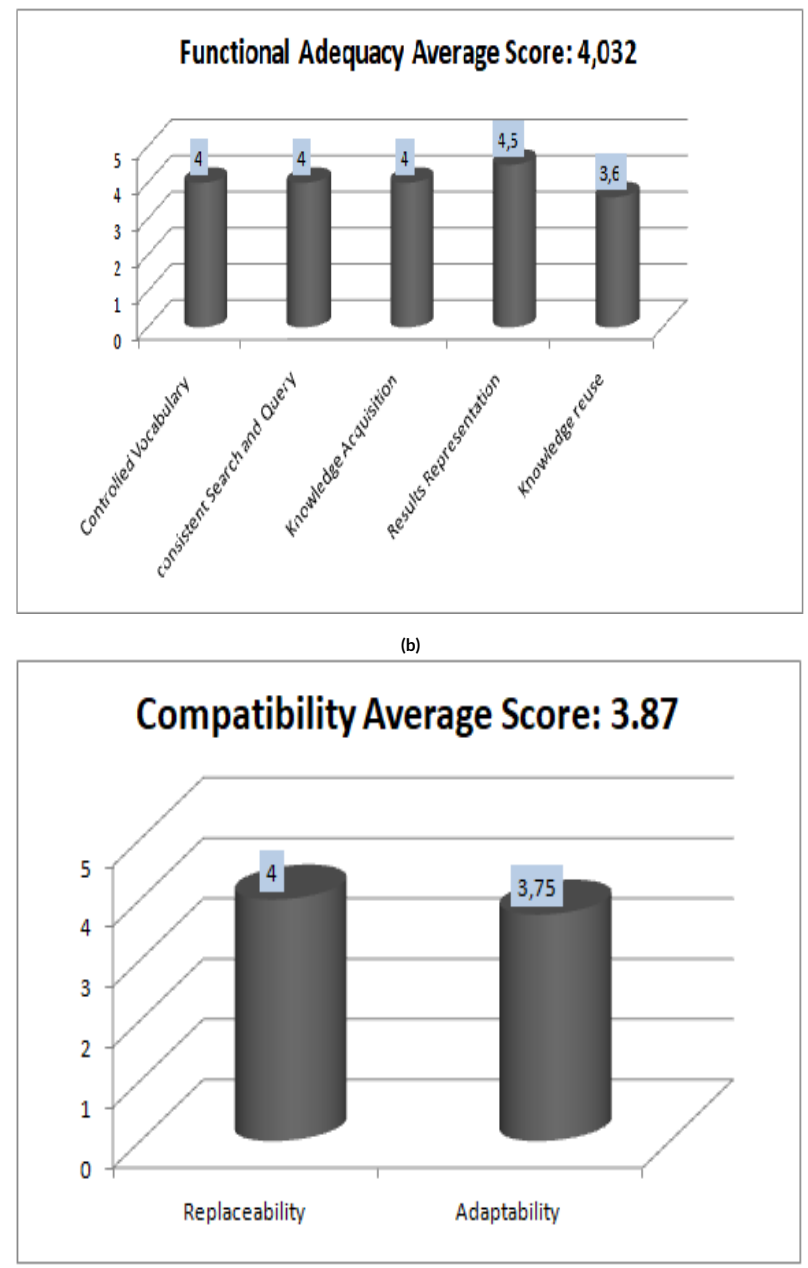

(d)

Operability Average Score: 4

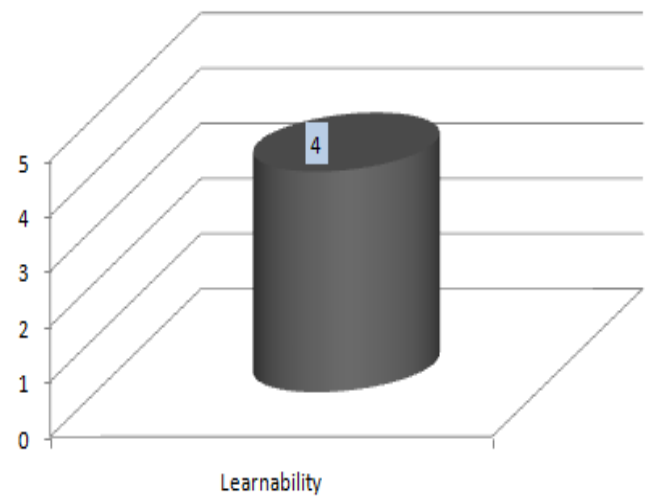

FIGURE 10 HealthloT- Ontology Evaluations using OQuaRE metrics.

$$
\begin{gathered}
\text { Precision }=\frac{T P}{(T P+F P)} \\
\text { Recall }=\frac{T P}{(T P+F N)}
\end{gathered}
$$




\section{F-measure $=\frac{2 * \text { Precision } * \text { Recall }}{\text { Precision }+ \text { Recall }}$}

The values of these measures reflect the relevance level of the inferred axioms by identifying the correct and the ambiguous ones.

For the experimentation we considered five datasets (temperature, blood pressure, blood glucose, cholesterol and heart rate) from physiobank 10 and we took 10 patients as sample. Therefore, for each dataset, we only took 1200 data records that correspond to the one-month duration for the monitoring of these patients ( 4 measurements per-day * 30 days * 10 patients). Consequently 6000 data records (1200*5) are stored in the HealthloT ontology. These datasets contains others informations such as the time and the location of these measures. Consequently, a total of 18300 instances (measures, time, location, symptoms, disease, drugs, food) were created to form the whole knowledge base of our clinical decision support system (loT-Medicare).

In fact, we have proposed diverse rules validated with health care experts ( 3 doctors) that helped us to calculate the reasoning phase performance of our knowledge base. Firstly, we proposed some rules taking into account just few contexts (age, and sex) (arround 20 rules) that help doctors for primary diagnosis. Secondly, we deeply focused on diverse contexts for advanced diagnosis process (arround 35 rules). Table 9 and Table 10 show the results of the correctly diagnosed patients with our system compared to those diagnosed manually (with domain experts), in the first and the second cases respectively.

TABLE 9 Comparing system classification with domain experts classification, in the primary diagnosis

\begin{tabular}{lll}
\hline & $\begin{array}{l}\text { Correctly diagnosed cases by } \\
\text { expert domain }\end{array}$ & $\begin{array}{l}\text { Incorrectly diagnosed cases by } \\
\text { expert domain }\end{array}$ \\
\hline $\begin{array}{l}\text { Correctly diagnosed cases by loT Medi- } \\
\text { care system }\end{array}$ & (2700) TP & (1310) FP \\
\hline $\begin{array}{l}\text { Incorrectly diagnosed cases by loT Medi- } \\
\text { care system }\end{array}$ & $(1030) \mathrm{FN}$ & (1020) TN \\
\hline
\end{tabular}

TABLE 10 Comparing system classification with domain experts classification with diverse contexts

\begin{tabular}{lll}
\hline & $\begin{array}{l}\text { Correctly diagnosed cases by } \\
\text { expert domain }\end{array}$ & $\begin{array}{l}\text { Incorrectly diagnosed cases by } \\
\text { expert domain }\end{array}$ \\
\hline $\begin{array}{l}\text { Correctly diagnosed cases by loT Medi- } \\
\text { care system }\end{array}$ & (3230) TP & (630) FP \\
\hline $\begin{array}{l}\text { Incorrectly diagnosed cases by loT Medi- } \\
\text { care system }\end{array}$ & (780) FN & (1360) TN \\
\hline
\end{tabular}

we recognized that taking into account context-aware reasoning gives a more precise and correct diagnosis and reduce adverse event that usually happens in the case of incorrect diagnosis.

Thereby, the performance of the developed system was improved as demonstrated by Figure 11.

\subsection{2 | Response Time of loT Medicare System}

This section was devoted to evaluate the effect of the data quantity and their contexts in the response time of the loT Medicare System. Thereby, the response time is defined as:

$$
T_{\text {rep }}=T_{\text {load }}+T_{\text {inf }}+T_{\text {quer } y}
$$

where $T_{\text {load }}$ is the time needed to load the ontology in the Drools engine, $T_{\text {inf }}$ is the time of the execution of the SWRL rules and $T_{\text {query }}$ determines the processing time of the SPARQL queries to display the results for the users.

\footnotetext{
${ }^{10} \mathrm{https} / / /$ physionet.org/physiobank/
} 


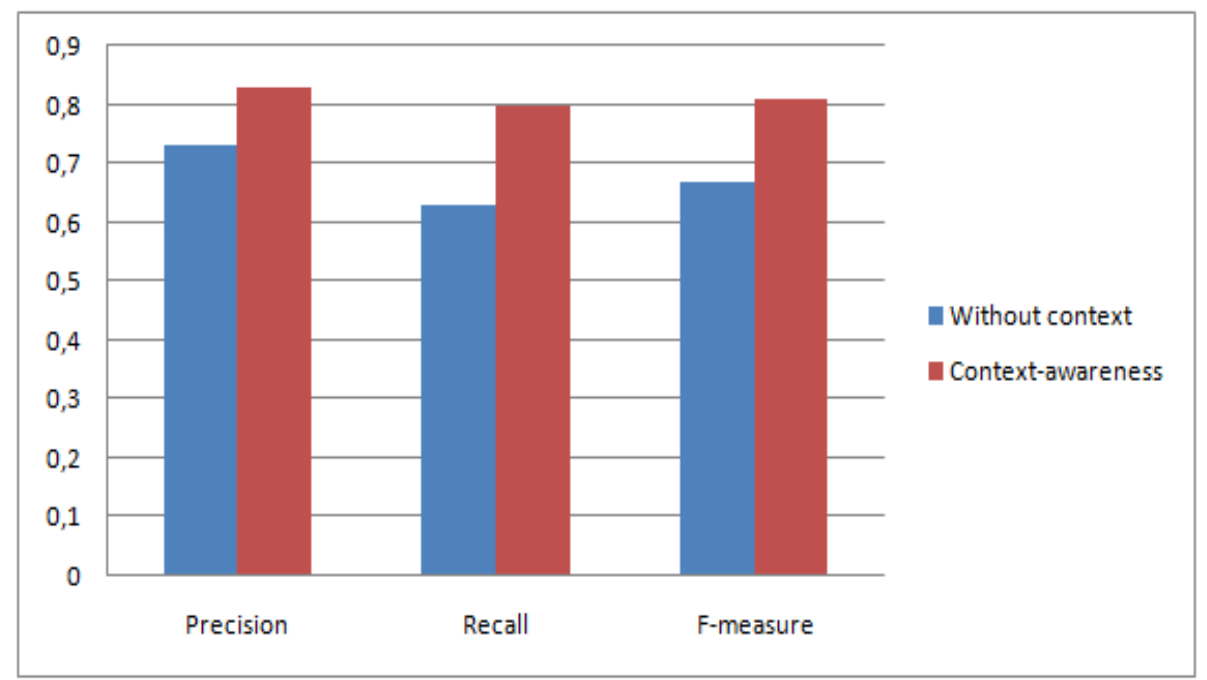

FIGURE 11 loT-Medicare system performance

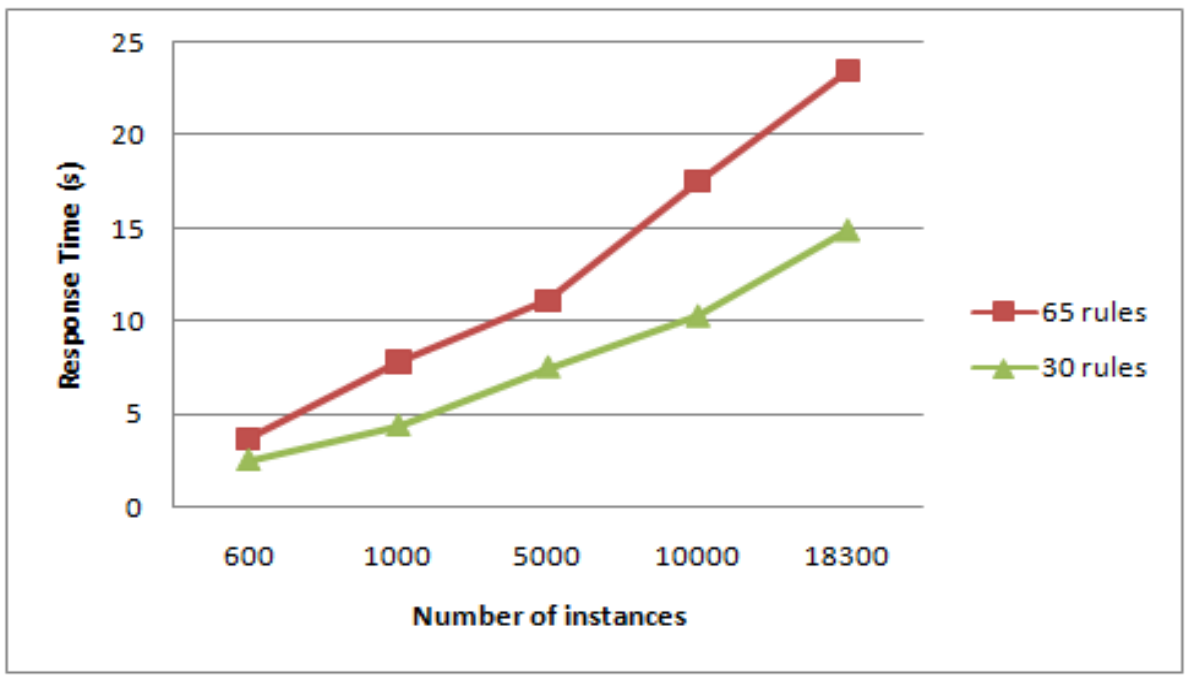

FIGURE 12 Response Time of loT Medicare System

HealthloT ontology is made up of more than 80 concepts, 100 object properties, and more than 90 data properties.

The first experimentation consists to set the number of rules to 30 and 65 for the second one. Then we vary the number of instances (600, 1000 , $5000,10000,18300$ ) in each experimentation.

The simulation is conducted with the following software platforms: Protege 5.0.1, JVM 1.8.1, Windows 7. The hardware platform is Intel Core $5 \mathrm{GHZ}$ CPU and 4GB RAM. In Figure 12, when the number of the stored data increases from 5000 to 18300 , the response time of the processing rules increases from 7,5 s to $14,87 \mathrm{~s}$ if we use only 30 rules. However, when we increase the number of rules to 65 , the response time is increased from 11,12 to $23,43 \mathrm{~s}$. Thus, the number of stored data and proposed rules can have a significant impact on the performance of the response time, especially if they involve complicated context reasoning.

\section{3 | Comparison with existing approaches}

In this section, we conduct a comparative study between the above mentioned works in section 3.3 and our work. The comparison is based on three main criteria as presented in Table 11. These criteria respond to our approach's goals. The coverage criterion identifies and highlights the reutilization of loT concepts and in order to evaluate the performance of our model, we try to compare it with other ones using their overall coverage 
as an assessment criterion. From this context, there are diverse referenced loT models such as loT-A Bauer et al. 2013 composed by 16 concepts, IoT-lite Bermudez-Edo et al. 2016 composed by 18 concepts, IOT-O Seydoux et al. 2016 that is composed by 28 concepts and FIESTA ontology that contains 26 concepts Agarwal et al. 2016. we have considered these ontologies and we calculated the coverage value of our ontology using the following equation.

$$
C=R C / T C
$$

Where $\mathrm{C}$ is the coverage, $\mathrm{RC}$ is the used concepts from the loT domain TC is the whole number of concepts in the loT model. The second criterion is about the reasoning task. Within this criterion, we verify what is the main objective of the proposed rules. Is it for device management or for disease management? The last criterion focuses on the adopted method for the evaluation of the proposed models.

TABLE 11 Comparison with existing works

\begin{tabular}{|c|c|c|c|c|c|c|c|c|c|c|c|}
\hline Reference & \multicolumn{4}{|c|}{ Coverage of loT concepts } & \multicolumn{5}{|c|}{ Reasoning } & \multicolumn{2}{|c|}{ Evaluation } \\
\hline $\begin{array}{l}\text { Forkan et } \\
\text { al. 2014 } \\
\end{array}$ & $\begin{array}{l}1 / 16= \\
6.2 \%\end{array}$ & $1 / 28=3 \%$ & $\begin{array}{l}1 / 18= \\
5.5 \%\end{array}$ & $1 / 26=3 \%$ & $+/-$ & Yes & No & No & Yes & $\begin{array}{l}\text { Response } \\
\text { time of } \\
\text { cloud node }\end{array}$ & No \\
\hline $\begin{array}{l}\text { Moreira et } \\
\text { al. 2018. }\end{array}$ & $\begin{array}{l}1 / 16= \\
6.2 \%\end{array}$ & $1 / 28=3 \%$ & $\begin{array}{l}1 / 18= \\
5.5 \%\end{array}$ & $1 / 26=3 \%$ & No & No & No & No & No & $\begin{array}{l}\text { Competency } \\
\text { Questions }\end{array}$ & No \\
\hline $\begin{array}{l}\text { Esposito } \\
\text { et al. } \\
2018 \\
\end{array}$ & $\begin{array}{l}1 / 16= \\
6.2 \%\end{array}$ & $1 / 28=3 \%$ & $\begin{array}{l}1 / 18= \\
5.5 \%\end{array}$ & $1 / 26=3 \%$ & No & Yes & No & Yes & Yes & No & No \\
\hline $\begin{array}{l}\text { Rubí and } \\
\text { Gondim } \\
2020\end{array}$ & $\begin{array}{l}1 / 16= \\
6.2 \%\end{array}$ & $1 / 28=3 \%$ & $\begin{array}{l}1 / 18= \\
5.5 \%\end{array}$ & $1 / 26=3 \%$ & No & Yes & No & No & Yes & No & No \\
\hline
\end{tabular}

From the comparative table our system differs from other approaches, by the following aspects:

- It is based on a generic knowledge base (HealthloT-ontology) that describes the amalgamation between the basic loT domain concepts and healthcare concepts. So that, it is useful for other applications in various contexts. It is better than the other systems which are based only on sensor 
device. Our ontology presents the best coverage value that ensures its reliability.

- Unlike other systems, our approach ensures configuration and management services for the deployed objects to guarantee a continuous and reliable patient monitoring.

- It suggests a whole diagnosis process which starts with data collection, diagnosis, risk prediction, treatment suggestion and then triggered alert. Most of these systems are proposed to accomplish a specific task, such as treatment or diagnosis. None of them has defined a whole diagnosis process unlike D. Chen et al. 2016) that focused on specific application (Hypertension disease).

- It is a context-aware system that treats diverse contexts by taking into account patients' states and medical devices.

- It offers user-friendly interfaces that can be used by three different users (patients, doctors, and administrators). However, the majority of other systems have been proposed for just one user; patients.

- Our system is flexible as it presents a graphical interface to doctors to be able to add or delete new patients and medical objects in the knowledge base.

- It provides patients with other various functions, such as the possibility to contact their doctors via a graphical interface which contains their healthcare information.

\section{0 | CONCLUSION AND OUTLOOK}

This paper proposed a semantic-based context-aware architecture approach for patient monitoring with MCOs. It was designed based on four fundamental phases such as the data collection and preprocessing phase, the semantic modeling phase, the analysis phase, and the implementation phase. The data collection and preprocessing phase considered two sources of data: data collected from MCO and data collected from medical records. The semantic modeling phase was interested in the representation of knowledge about the MCO, the monitored patient, and their contexts. The resulted semantic model called "HealthloT Ontology" was exploited by defining diverse rules based on the SWRL language in the analysis phase. These rules were suggested for two main objectives: the configuration and the management of the employed objects and the patient state diagnosis and decision making taking into account the alterable context. The implementation phase was focused on the development of an loT Medicare system for patient monitoring, which integrated both the proposed knowledge base (HealthloT-O) and the rule base in order to provide diverse services for end-users (doctor, patient, and administrator) according to its deployment context. The evaluation of our approach focused on two objectives: a technical evaluation that interested in the semantic quality of HealthloT ontology and a functional evaluation that focused on the reasoning performance and the response time of the loT Medicare system.

The current work offers many challenges and different perspectives that we plan to address them in future works. Firstly, we will focus on extending and improving our approach firstly, by implementing a real use case and using health standards such as FHIR, ISO TS for health sensor data representation. In addition, we will extract clinical information automatically from electronic sources (the EHR) by applying the NLP technics and deep learning. Therefore, will focus on the alignment of our ontology with other domain ontology in order to enhance its capacity on accommodating different application domains. Secondly, we aim to evaluate the capacity of our approach in the monitoring of patients which suffers from Coronavirus.

In addition, we aim to propose and to implement an intelligent solution to optimize the use of the ontology instances to be then smartly processed and analyzed. This solution will be able to define a direct and seamless interpretation of the knowledge base and the system without using the inference engine. By this way, we ensure that the more accurate used knowledge, the more understandable and reasonable system we can define. In addition, we will focus on the scalability challenge of the loT-Medicare system in order to be capable to manage the possible huge quantity of data accurately. In this context, Big data technology can be exploited as a solution.

\section{References}

Aborokbah, M. M., Al-Mutairi, S., Sangaiah, A. K., \& Samuel, O. W. (2018). Adaptive context aware decision computing paradigm for intensive health care delivery in smart cities-a case analysis. Sustainable cities and society, 41, 919-924.

Abu-Elkheir, M., Hayajneh, M., \& Ali, N. A. (2013). Data management for the internet of things: Design primitives and solution. Sensors, 13(11), 15582-15612. 
Agarwal, R., Fernandez, D. G., Elsaleh, T., Gyrard, A., Lanza, J., Sanchez, L., ... Issarny, V. (2016). Unified iot ontology to enable interoperability and federation of testbeds. In Internet of things (wf-iot), 2016 ieee 3rd world forum on (pp. 70-75).

Alirezaie, M., Renoux, J., Köckemann, U., Kristoffersson, A., Karlsson, L., Blomqvist, E., ... Loutf, A. (2017). An ontology-based context-aware system for smart homes: E-care@ home. Sensors, 17(7), 1586.

Angsuchotmetee, C., Chbeir, R., \& Cardinale, Y. (2018). Mssn-onto: An ontology-based approach for flexible event processing in multimedia sensor networks. Future Generation Computer Systems.

Arfaoui, A., Kribeche, A., \& Senouci, S.-M. (2019). Context-aware anonymous authentication protocols in the internet of things dedicated to e-health applications. Computer Networks, 159, 23-36.

Bai, L., Yang, D., Wang, X., Tong, L., Zhu, X., Bai, C., \& Powell, C. A. (2020). Chinese experts' consensus on the internet of things-aided diagnosis and treatment of coronavirus disease 2019. Clinical eHealth.

Baloch, Z., Shaikh, F. K., \& Unar, M. A. (2018). A context-aware data fusion approach for health-iot. International Journal of Information Technology, 10(3), 241-245.

Barbosa, J., Tavares, J., Cardoso, I., Alves, B., \& Martini, B. (2018). Trailcare: An indoor and outdoor context-aware system to assist wheelchair users. International Journal of Human-Computer Studies, 116, 1-14.

Bauer, M., Boussard, M., Bui, N., De Loof, J., Magerkurth, C., Meissner, S., ... Walewski, J. W. (2013). lot reference architecture. In Enabling things to talk (pp. 163-211). Springer, Berlin, Heidelberg.

Bendadouche, R., Roussey, C., De Sousa, G., Chanet, J.-P., \& Hou, K. M. (2012). Extension of the semantic sensor network ontology for wireless sensor networks: The stimulus-wsnnode-communication pattern. In Proceedings of the 5th international conference on semantic sensor networks-volume 904 (pp. 49-64).

Bermudez-Edo, M., Elsaleh, T., Barnaghi, P., \& Taylor, K. (2016). lot-lite: a lightweight semantic model for the internet of things. In 2016 intl ieee conferences on ubiquitous intelligence \& computing, advanced and trusted computing, scalable computing and communications, cloud and big data computing, internet of people, and smart world congress (uic/atc/scalcom/cbdcom/iop/smartworld) (pp. 90-97).

Berners-Lee, T., Hendler, J., \& Lassila, O. (2001). The semantic web. Scientific american, 284(5), 34-43.

Cai, H., Da Xu, L., Xu, B., Xie, C., Qin, S., \& Jiang, L. (2014). lot-based configurable information service platform for product lifecycle management. IEEE Transactions on Industrial Informatics, 10(2), 1558-1567.

Cappon, G., Acciaroli, G., Vettoretti, M., Facchinetti, A., \& Sparacino, G. (2017). Wearable continuous glucose monitoring sensors: A revolution in diabetes treatment. Electronics, 6(3), 65.

Chatterjee, S., Byun, J., Dutta, K., Pedersen, R. U., Pottathil, A., \& Xie, H. (2018). Designing an internet-of-things (iot) and sensor-based in-home monitoring system for assisting diabetes patients: iterative learning from two case studies. European Journal of Information Systems, 27(6), 670-685.

Chegini, H., \& Mahanti, A. (2019). A framework of automation on context-aware internet of things (iot) systems. In Proceedings of the 12 th ieee/acm international conference on utility and cloud computing companion (pp. 157-162).

Chen, D., Jin, D., Goh, T.-T., Li, N., \& Wei, L. (2016). Context-awareness based personalized recommendation of anti-hypertension drugs. Journal of medical systems, 40(9), 202.

Chen, Y., Zhou, J., \& Guo, M. (2016). A context-aware search system for internet of things based on hierarchical context model. Telecommunication Systems, 62(1), 77-91.

Compton, M., Barnaghi, P., Bermudez, L., GarcíA-Castro, R., Corcho, O., Cox, S., ... others (2012). The ssn ontology of the w3c semantic sensor network incubator group. Web semantics: science, services and agents on the World Wide Web, 17, 25-32.

Dey, A. K., Abowd, G. D., \& Salber, D. (2001). A conceptual framework and a toolkit for supporting the rapid prototyping of context-aware applications. Human-Computer Interaction, 16(2-4), 97-166.

Duque-Ramos, A., Boeker, M., Jansen, L., Schulz, S., Iniesta, M., \& Fernández-Breis, J. T. (2014). Evaluating the good ontology design guideline (goodod) with the ontology quality requirements and evaluation method and metrics (oquare). PloS one, 9(8), e104463.

Duque-Ramos, A., Fernández-Breis, J. T., Iniesta, M., Dumontier, M., Aranguren, M. E., Schulz, S., ... Stevens, R. (2013). Evaluation of the oquare framework for ontology quality. Expert Systems with Applications, 40(7), 2696-2703.

Duque-Ramos, A., Fernández-Breis, J. T., Stevens, R., Aussenac-Gilles, N., et al. (2011). Oquare: A square-based approach for evaluating the quality of ontologies. Journal of Research and Practice in Information Technology, 43(2), 159.

Esposito, M., Minutolo, A., Megna, R., Forastiere, M., Magliulo, M., \& De Pietro, G. (2018). A smart mobile, self-configuring, context-aware architecture for personal health monitoring. Engineering Applications of Artificial Intelligence, 67, 136-156.

Fernandez, F., \& Pallis, G. C. (2014). Opportunities and challenges of the internet of things for healthcare: Systems engineering perspective. In Wireless mobile communication and healthcare (mobihealth), 2014 eai 4th international conference on (pp. 263-266).

Forkan, A., Khalil, I., \& Tari, Z. (2014). Cocamaal: A cloud-oriented context-aware middleware in ambient assisted living. Future Generation Computer 
Systems, 35, 114-127.

Gatouillat, A., Badr, Y., Massot, B., \& Sejdić, E. (2018). Internet of medical things: A review of recent contributions dealing with cyber-physical systems in medicine. IEEE Internet of Things Journal.

Goldberger, A. L., Amaral, L. A., Glass, L., Hausdorff, J. M., Ivanov, P. C., Mark, R. G., ... Stanley, H. E. (2000). Physiobank, physiotoolkit, and physionet: components of a new research resource for complex physiologic signals. circulation, 101(23), e215-e220.

Gonçalves, B., Zamborlini, V., \& Guizzardi, G. (2009). An ontological analysis of the electrocardiogram. Electronic Journal of Communication, Information and Innovation in Health, 3, 1-26.

Gupta, A. K., Chakraborty, C., \& Gupta, B. (2019). Monitoring of epileptical patients using cloud-enabled health-iot system. Traitement du Signal, 36(5), 425-431.

Hobbs, J. R., \& Pan, F. (2006). Time ontology in owl. W3C working draft, 27, 133.

Islam, S. R., Kwak, D., Kabir, M. H., Hossain, M., \& Kwak, K.-S. (2015). The internet of things for health care: a comprehensive survey. IEEE Access, 3 , 678-708.

ISO/IEC. (2008). Iso/iec 25012: 2008, software engineering-software product quality requirements and evaluation (square)-data quality model. International Organisation for Standardisation Geneva.

Jabeen, F., Maqsood, M., Ghazanfar, M. A., Aadil, F., Khan, S., Khan, M. F., \& Mehmood, I. (2019). An iot based efficient hybrid recommender system for cardiovascular disease. Peer-to-Peer Networking and Applications, 12(5), 1263-1276.

Janowicz, K., Haller, A., Cox, S. J., Le Phuoc, D., \& Lefrancois, M. (2018). Sosa: A lightweight ontology for sensors, observations, samples, and actuators. Journal of Web Semantics.

Jayatilleka, I., \& Halgamuge, M. N. (2020). Internet of things in healthcare: Smart devices, sensors, and systems related to diseases and health conditions. In Real-time data analytics for large scale sensor data (pp. 1-35). Elsevier.

Khan, M. A. (2020). An iot framework for heart disease prediction based on mdenn classifier. IEEE Access, 8, 34717-34727.

Kim, J., Campbell, A. S., \& Wang, J. (2018). Wearable non-invasive epidermal glucose sensors: A review. Talanta, 177, $163-170$.

Lasierra, N., Alesanco, A., O'Sullivan, D., \& García, J. (2013). An autonomic ontology-based approach to manage information in home-based scenarios: From theory to practice. Data \& Knowledge Engineering, 87, 185-205.

Liu, F., Chen, Z., \& Wang, J. (2018). Intelligent medical iot system based on wsn with computer vision platforms. Concurrency and Computation: Practice and Experience, e5036.

Ma, M., Wang, P., \& Chu, C.-H. (2014). Ontology-based semantic modeling and evaluation for internet of things applications. In 2014 ieee international conference on internet of things (ithings), and ieee green computing and communications (greencom) and ieee cyber, physical and social computing (cpscom) (pp. 24-30).

Meigal, A. Y., Korzun, D. G., Gerasimova-Meigal, L. I., Borodin, A. V., \& Zavyalova, Y. V. (2019). Ambient intelligence at-home laboratory for human everyday life. International Journal of Embedded and Real-Time Communication Systems (IJERTCS), 10(2), 117-134.

Mishra, S. S., \& Rasool, A. (2019). lot health care monitoring and tracking: A survey. In 2019 3rd international conference on trends in electronics and informatics (icoei) (pp. 1052-1057).

Moreira, J., Pires, L. F., van Sinderen, M., \& Daniele, L. (2018). Saref4health: lot standard-based ontology-driven healthcare systems. In Fois (pp. 239-252).

Motik, B., Patel-Schneider, P. F., Parsia, B., Bock, C., Fokoue, A., Haase, P., ... others (2009). Owl 2 web ontology language: Structural specification and functional-style syntax. W3C recommendation, 27(65), 159.

Müller, H., Cabral, L., Morshed, A., \& Shu, Y. (2013). From restful to sparql: a case study on generating semantic sensor data. In Proceedings of the 6th international conference on semantic sensor networks (Vol. Volume 1063, pp. 51-66).

Organization, W. H., et al. (2016). Global report on diabetes. World Health Organization.

Perera, C., Zaslavsky, A., Christen, P., Compton, M., \& Georgakopoulos, D. (2013). Context-aware sensor search, selection and ranking model for internet of things middleware. In Mobile data management (mdm), 2013 ieee 14th international conference on (Vol. 1, pp. 314-322).

Poulovassilis, A., Papamarkos, G., \& Wood, P. T. (2006). Event-condition-action rule languages for the semantic web. In International conference on extending database technology (pp. 855-864).

Puri, V., Kumar, R., Le, D. N., Jagdev, S. S., \& Sachdeva, N. (2020). Biosenhealth 2.0-a low-cost, energy-efficient internet of things-based blood glucose monitoring system. In Emergence of pharmaceutical industry growth with industrial iot approach (pp. 305-324). Elsevier.

Rahman, H., Rahmani, R., \& Kanter, T. (2017). Multi-modal context-aware reasoner (can) at the edge of iot. In Ant/seit (pp. 335-342).

Rhayem, A., Mhiri, M. B. A., \& Gargouri, F. (2017). Healthiot ontology for data semantic representation and interpretation obtained from medical connected objects. In Computer systems and applications (aiccsa), 2017 ieee/acs 14th international conference on (pp. 1470-1477).

Rhayem, A., Mhiri, M. B. A., Salah, M. B., \& Gargouri, F. (2017). Ontology-based system for patient monitoring with connected objects. Procedia Computer Science, 112, 683-692. 
Rubí, J. N. S., \& Gondim, P. R. d. L. (2020). Interoperable internet of medical things platform for e-health applications. International Journal of Distributed Sensor Networks, 16(1), 1550147719889591.

Santos, V., Trujillo, M., Portilla, K., \& Rosales, A. (2019). Accessible ehealth system for heart rate estimation. In The international conference on advances in emerging trends and technologies (pp. 260-269).

Senior, M. (2014). Novartis signs up for google smart lens. Nature Publishing Group.

Seydoux, N., Drira, K., Hernandez, N., \& Monteil, T. (2016). lot-o, a core-domain iot ontology to represent connected devices networks. In Knowledge engineering and knowledge management: 20th international conference, ekaw 2016, bologna, italy, november 19-23, 2016, proceedings 20 (pp. 561 576).

Smith, B., Ashburner, M., Rosse, C., Bard, J., Bug, W., Ceusters, W., ... others (2007). The obo foundry: coordinated evolution of ontologies to support biomedical data integration. Nature biotechnology, 25(11), 1251.

Sood, S. K., \& Mahajan, I. (2018). lot-fog-based healthcare framework to identify and control hypertension attack. IEEE Internet of Things Journal, 6(2), 1920-1927.

Studer, R., Benjamins, V. R., \& Fensel, D. (1998). Knowledge engineering: principles and methods. Data \& knowledge engineering, 25(1-2), $161-197$.

Topouchian, J., Agnoletti, D., Blacher ${ }^{1}$, J., Youssef, A., Chahine, M. N., Ibanez, I., ... Asmar, R. (2014). Validation of four devices: Omron m6 comfort, omron hem-7420, withings bp-800, and polygreen kp-7670 for home blood pressure measurement according to the european society of hypertension international protocol. Vascular health and risk management, 10, 33.

Tung, C. E., Su, D., Turakhia, M. P., \& Lansberg, M. G. (2015). Diagnostic yield of extended cardiac patch monitoring in patients with stroke or tia. Frontiers in neurology, 5, 266.

Turcu, C., \& Turcu, C. (2019). Improving the quality of healthcare through internet of things. arXiv preprint arXiv:1903.05221.

Varatharajan, R., Manogaran, G., Priyan, M. K., \& Sundarasekar, R. (2018). Wearable sensor devices for early detection of alzheimer disease using dynamic time warping algorithm. Cluster Computing, 21(1), 681-690.

Wang, H.-C., \& Lee, A.-R. (2015). Recent developments in blood glucose sensors. Journal of food and drug analysis, 23(2), 191-200.

Wannous, R., Malki, J., Bouju, A., \& Vincent, C. (2013). Modelling mobile object activities based on trajectory ontology rules considering spatial relationship rules. In Modeling approaches and algorithms for advanced computer applications (pp. 249-258). Springer.

Yürür, Ö., Liu, C. H., Sheng, Z., Leung, V. C., Moreno, W., \& Leung, K. K. (2016). Context-awareness for mobile sensing: A survey and future directions. IEEE Communications Surveys \& Tutorials, 18(1), 68-93.

Conflict of interest The authors declare no potential conflict of interests. 